\title{
On $2 k$-Variable Symmetric Boolean Functions with Maximum Algebraic Immunity $k$
}

\author{
Hui Wang, Jie Peng, Yuan Li, and Haibin Kan
}

\begin{abstract}
Algebraic immunity of Boolean function $f$ is defined as the minimal degree of a nonzero $g$ such that $f g=0$ or $(f+1) g=0$. Given a positive even integer $n$, it is found that the weight distribution of any $n$-variable symmetric Boolean function with maximum algebraic immunity $\frac{n}{2}$ is determined by the binary expansion of $n$. Based on the foregoing, all $n$-variable symmetric Boolean functions with maximum algebraic immunity are constructed. The amount is $(2 \mathbf{w t}(n)+1) 2^{\left\lfloor\log _{2} n\right\rfloor}$.
\end{abstract}

Index Terms-Algebraic attack, algebraic immunity, symmetric Boolean function.

\section{INTRODUCTION}

A LGEBRAIC attacks have received much attention in cryptographic analyzing stream and block cipher systems [1], [6], [18], which try to recover the secret key by solving overdefined systems of multivariate equations. Therefore, algebraic immunity (AI), a new cryptographic property for designing Boolean functions, was proposed by W. Meier et al. 14]. Algebraic immunity of the Boolean function used in a cryptosystem should be high enough to resist algebraic attacks. The upper bound of the algebraic immunity of an $n$ variable Boolean function is $\left\lceil\frac{n}{2}\right\rceil$ [6], [14]. Several theoretical constructions of Boolean functions with optimal AI have been presented in the literature [5], 8], [10], [15].

Symmetric Boolean functions are of great interest from a cryptographic point of view. An $n$-variable symmetric Boolean function can be identified by an $(n+1)$-bit vector, so symmetric Boolean functions have smaller hardware size than average Boolean functions. They allow the computation of values for functions with more variables than general ones. For this reason, symmetric Boolean functions have been paid particular attention.

For an odd integer $n$, Dalai et al. showed that a Boolean function with maximum AI should be balanced [8]. In [13], it was proved that the majority function $\mathrm{Maj}_{n}$ and its complement $\mathrm{Maj}_{n}+1$ are the only two trivially balanced symmetric Boolean functions with maximum AI. It also has been proven that the number of symmetric Boolean functions with maximum AI is exactly two [16].

For the case where $n$ is even, the situation becomes very complicated. A few classes of even-variable symmetric

H. Wang is with Shanghai Key Lab of Intelligent Information Processing, School of Computer Science, Fudan University, Shanghai 200433, P. R. China (e-mail: 1011024022@fudan.edu.cn).

J. Peng is with School of Mathematics and Statistics, Huazhong Normal University, Wuhan 430079, P. R. China (e-mail: jiepeng@mail.ccnu.edu.cn).

Y. Li and H. Kan are with Shanghai Key Lab of Intelligent Information Processing, School of Computer Science, Fudan University, Shanghai 200433, P. R. China (e-mails: 07300720173@fudan.edu.cn; hbkan@fudan.edu.cn).
Boolean functions with maximum AI have been constructed in [15], [3]. However, only the number and form of $2^{m}$-variable symmetric Boolean functions with maximum algebraic immunity have been solved by introducing the weight support technique [10]. This method has also been used to determine the number of $\left(2^{m}+1\right)$-variable symmetric Boolean functions with submaximum algebraic immunity $2^{m-1}$ [12].

In this paper, we first study the weight distribution of those $n$-variable symmetric Boolean functions achieving maximum algebraic immunity with $n$ even. We find that the set $N=\{0,1, \ldots, n\}$ can be divided into some particular subsets according to the binary expansion of $n$, on which the Boolean functions should be constant. Meanwhile, the values of the functions on these subsets should satisfy some strict conditions. Furthermore, we continue to prove that all the symmetric Boolean functions constructed following the above laws indeed achieve maximum algebraic immunity. Thus, we construct all the even-variable symmetric Boolean functions with maximum algebraic immunity. The number of these functions and their corresponding hamming weights are also obtained.

The organization of the paper is as follows. In the following section, we present some basic notations and knowledge about Boolean functions. In section 3, we obtain some necessary conditions for an even-variable symmetric Boolean function to reach maximum algebraic immunity. In the next two sections, we prove that these conditions are sufficient. The main theorem of this paper is given in section 6 . Section 7 concludes the paper.

\section{PRELIMINARIES}

Let $\mathrm{F}_{2}^{n}$ be the $n$-dimensional vector space over the finite field $\mathrm{F}_{2}$, and $e_{0}^{n}, e_{1}^{n}, \ldots, e_{n-1}^{n}$ be its normal basis,

$e_{0}^{n}=(1,0, \ldots, 0), e_{1}^{n}=(0,1, \ldots, 0), \ldots, e_{n-1}^{n}=(0,0, \ldots, 1)$.

The superscript $n$ may be omitted if there is no confusion.

An $n$-variable Boolean function is a function from $\mathrm{F}_{2}^{n}$ into $\mathrm{F}_{2}$. Let $B_{n}$ be the ring of Boolean functions on $n$ variables $x_{1}, x_{2}, \ldots, x_{n}$, then

$$
\mathrm{B}_{n}=\mathrm{F}_{2}\left[x_{1}, \ldots, x_{n}\right] /\left(x_{1}^{2}+x_{1}, \ldots, x_{n}^{2}+x_{n}\right),
$$

and every $f \in \mathrm{B}_{n}$ can be uniquely written in the polynomial form $f=\sum_{I \in F_{2}^{n}} a_{I} x^{I}$, where $x^{I}=x_{1}^{i_{1}} x_{2}^{i_{2}} \cdots x_{n}^{i_{n}}$, which is called the algebraic normal form (ANF) of $f$. The algebraic degree of $f$, denoted by $\operatorname{deg}(f)$, is the degree of the polynomial. 
For $f \in \mathrm{B}_{n}$, the algebraic immunity of $f$, denoted by $\operatorname{AI}(f)$ is defined to be the lowest degree of nonzero annihilators of $f$ or $f+1$, i.e.,

$$
\mathrm{AI}(f)=\min \{\operatorname{deg}(g) \mid g \neq 0, f g=0 \text { or }(f+1) g=0\} .
$$

Two Boolean functions $f$ and $g$ are called to be affine equivalent if there exist $A \in \mathrm{GL}_{n}\left(\mathrm{~F}_{2}\right)$ and $\varphi \in \mathrm{F}_{2}^{n}$ such that $g(x)=f(x A+\varphi)$. Clearly, algebraic degree and algebraic immunity are both affine invariant.

Let $\alpha=\left(\alpha_{1}, \alpha_{2}, \ldots, \alpha_{n}\right) \in \mathrm{F}_{2}^{n}$, the Hamming weight of $\alpha$, denoted by $\mathrm{wt}(\alpha)$, is the number of 1 's in $\left\{\alpha_{1}, \alpha_{2}, \ldots, \alpha_{n}\right\}$. For an integer $i>0$ with 2-adic expansion $i=\sum_{j=0}^{m} i_{j} 2^{j}$, wt $(i)$ represents the Hamming weight of its binary expansion $\left(i_{m}, \ldots, i_{1}, i_{0}\right)_{2}$.

Let $\operatorname{supp}(f)=\left\{x \in \mathrm{F}_{2}^{n} \mid f(x)=1\right\}$, the cardinality of $\operatorname{supp}(f)$, denoted by $\operatorname{wt}(f)$, is called the Hamming weight of $f$. We say that an $n$-variable Boolean function $f$ is balanced if $\operatorname{wt}(f)=2^{n-1}$. The weight support [10] of $f$, denoted by $\mathrm{WS}(f)$, is defined to be

$$
\mathrm{WS}(f)=\{i \mid \exists x \in \operatorname{supp}(f) \text { such that } \operatorname{wt}(x)=i\} .
$$

We will use $P_{b}$ to represent the polynomial

$$
\left(x_{1}+x_{2}\right)\left(x_{3}+x_{4}\right) \cdots\left(x_{2 b-1}+x_{2 b}\right) .
$$

Note that $P_{b}$ is a $(2 b)$-variable polynomial with $\operatorname{deg}\left(P_{b}\right)=b$ and $\operatorname{WS}\left(P_{b}\right)=\{b\}$.

A Boolean function $f$ is symmetric if its output is invariant under any permutation of its input bits, i.e.,

$$
f\left(x_{1}, x_{2}, \ldots, x_{n}\right)=f\left(x_{\sigma(1)}, x_{\sigma(2)}, \ldots, x_{\sigma(n)}\right)
$$

for any permutation $\sigma$ of $\{1,2, \ldots, n\}$.

Let $\mathrm{SB}_{n}$ be the ring of symmetric Boolean functions on $n$ variables $x_{1}, x_{2}, \ldots, x_{n}$, then every $f \in \mathrm{SB}_{n}$ can be represented by a vector

$$
v_{f}=\left(v_{f}(0), v_{f}(1), \ldots, v_{f}(n)\right) \in \mathrm{F}_{2}^{n+1},
$$

where the component $v_{f}(i)$ represents the function value for vectors of weight $i$. The vector $v_{f}$ is called the simplified value vector (SVV) of $f$. If $f \in \mathrm{SB}_{n}$ and $f^{\prime}\left(x_{1}, \ldots, x_{n}\right)=$ $f\left(x_{1}+1, \ldots, x_{n}+1\right)$, then $f^{\prime} \in \mathrm{SB}_{n}$ is affine equivalent to $f$, and $v_{f^{\prime}}(i)=v_{f}(n-i)$, for any $0 \leq i \leq n$.

On the other hand, the ANF of $f$ can be written as

$$
f\left(x_{1}, x_{2}, \ldots, x_{n}\right)=\sum_{i=0}^{n} \lambda_{f}(i) \sigma_{i}^{n},
$$

where $\sigma_{i}^{n}$ is the homogeneous symmetric Boolean function on $\mathrm{n}$ variables which consists of all the terms of degree $i$. The vector

$$
\lambda_{f}=(\lambda(0), \lambda(1), \ldots, \lambda(n)) \in \mathrm{F}_{2}^{n+1}
$$

is called the simplified algebraic normal form (SANF) vector of $f$. Both $v_{f}$ and $\lambda_{f}$ can be regarded as mappings from $\{0,1, \ldots, n\}$ to $\mathrm{F}_{2}$.

Let $a$ and $b$ be two nonnegative integers with 2-adic expansions

$$
a=\sum_{j=0}^{m} a_{j} 2^{j}, b=\sum_{j=0}^{m} b_{j} 2^{j} .
$$

We say $a \preceq b$ if $a_{j} \leq b_{j}$ for any $0 \leq j \leq m$ and $a \prec b$ if $a \preceq b$ and $a \neq b$. Using the Lucas formula which states that $\left(\begin{array}{l}a \\ b\end{array}\right)=1 \in \mathrm{F}_{2}$ if and only if $b \preceq a$, we can derive the following two lemmas:

Lemma 2.1: [4] Let $f$ be an $n$-variable symmetric Boolean function. Then

$$
\lambda_{f}(i)=\sum_{k \preceq i} v_{f}(k), v_{f}(i)=\sum_{k \preceq i} \lambda_{f}(k) .
$$

Lemma 2.2: 4] For $\ell \geq 1$, suppose $f \in \mathrm{SB}_{n}$ and $\operatorname{deg}(f)<$ $2^{\ell}$, then $v_{f}$ has period $2^{\ell}$, which means $v_{f}(i)=v_{f}\left(i+2^{\ell}\right)$ for $0 \leq i \leq n-2^{\ell}$.

Lemma 2.2 can be derived easily from Lemma 2.1 .

Lemma 2.3: [9] Let $n=2 k$ and $G_{n}$ be an $n$-variable symmetric Boolean function. If its simplified value vector $v_{G_{n}}$ satisfies

$$
v_{G_{n}}(i)= \begin{cases}0, & \text { for } i \leq k, \\ 1, & \text { for } i>k,\end{cases}
$$

then $\operatorname{AI}\left(G_{n}\right)=k$. Function $G_{n}$ is called the majority function.

Lemma 2.4: [10] Suppose that $n \geq 2$ and $f \in \mathrm{SB}_{n}$. If there exists $0 \neq g \in \mathrm{B}_{n}$, such that $f g=0$, then there exists $b$, $0 \leq b \leq\left\lfloor\frac{n}{2}\right\rfloor$ and $0 \neq h\left(x_{2 b+1}, \ldots, x_{n}\right) \in \mathrm{SB}_{n-2 b}, \operatorname{deg}(h) \leq$ $\operatorname{deg}(g)-b$, such that $f h P_{b}=0$.

Lemma 2.5: Suppose $n=2 k$ and $f \in \mathrm{SB}_{n}$. If $\mathrm{AI}(f)=k$, then $\operatorname{wt}\left(v_{f}\right) \in\{k, k+1\}$.

Proof: It is sufficient to prove that when $\operatorname{wt}\left(v_{f}\right)<k$ or $\operatorname{wt}(f)>k+1, f$ or $f+1$ has a nonzero symmetric annihilator with degree less than $k$. Without loss of generality, we consider that $\operatorname{wt}\left(v_{f}\right)<k$. Otherwise, we can replace $f$ by $f+1$.

Let $g=\sum_{i=0}^{k-1} \lambda_{g}(i) \sigma_{i}$ be a symmetric annihilator of $f$. Hence, $f g=0$ if and only if for all $v_{f}(i)=1, v_{g}(i)=0$ holds. Thus, by Lemma $2.1, \operatorname{wt}\left(v_{f}\right)$ equations on $k$ variables $\lambda_{g}(0), \ldots, \lambda_{g}(k-1)$ are obtained, where the number of equations is less than the number of unknowns. Therefore, at least one nonzero solution exists, which implies the existence of such an annihilator.

\section{Necessary Conditions for EVEn-VARIABle} Symmetric BoOlean Functions With MaXimum AI

We always assume $n=2 k$. In this section, we will present the constraints on the simplified value vector for an $n$-variable symmetric Boolean function $f$ with maximum algebraic immunity $k$ step by step. First, we present Lemma 3.1 and Theorem 3.1, where Lemma 3.1 is a special case of Theorem 3.1. According to Lemma 3.1 and Theorem 3.1, several notations and definitions are given. Based on them, we present Corollary 3.1, Theorem 3.2, Theorem 3.3, and Theorem 3.4, which are the main results of this section. Theorem 3.5 concludes this section by showing two classes of symmetric Boolean functions satisfying all the necessary conditions. The following lemma is very important. 
Lemma 3.1: Let $n=2^{p+1} \mu$ with $p, \mu \geq 1$, and $f \in \mathrm{SB}_{n}$. If $\mathrm{AI}(f)=k$, then

$$
v_{f}\left(2^{p} \mu-2^{p} i+2^{p-1}\right)=v_{f}\left(2^{p} \mu+2^{p} j-2^{p-1}\right)+1
$$

for any $1 \leq i, j \leq \mu$.

Proof: We will prove this theorem by induction on parameter $\mu$.

Basis: When $\mu=1$, it is true due to Theorem 2.2 of [17.

Induction: Assuming the theorem is true for $\mu=\ell \geq 1$, we claim that it is also true for $\mu=\ell+1$. Now, let $n=$ $2 k=2^{p+1}(\ell+1)$ and $f \in \mathrm{SB}_{n}$ with $\mathrm{AI}(f)=k=2^{p}(\ell+1)$. We will prove that the Boolean function $f$ satisfies $v_{f}\left(2^{p}(\ell+\right.$ 1) $\left.-2^{p} i+2^{p-1}\right)=v_{f}\left(2^{p}(\ell+1)+2^{p} j-2^{p-1}\right)+1$ for any $1 \leq i, j \leq \ell+1$ in the following four steps.

In step 1 , we prove (1) for $1 \leq i, j \leq \ell$; in step 2 , for $1 \leq j \leq \ell$ and $i=\ell+1$; in step 3 , for $1 \leq i \leq \ell$ and $j=\ell+1$; and in step 4 , for $i=\ell+1$ and $j=\ell+1$.

Step 1 Assume to the contrary that $v_{f}\left(2^{p}(\ell+1)-2^{p} i+\right.$ $\left.2^{p-1}\right)=v_{f}\left(2^{p}(\ell+1)+2^{p} j-2^{p-1}\right)$ for some $1 \leq i, j \leq$ $\ell$, letting $f^{\prime} \in \mathrm{SB}_{2^{p+1} \ell}$ on variables $x_{2^{p+1}+1}, x_{2^{p+1}+2}, \ldots$, $x_{2^{p+1}(\ell+1)}$, be defined as

$$
v_{f^{\prime}}=\left(v_{f}\left(2^{p}\right), v_{f}\left(2^{p}+1\right), \ldots, v_{f}\left(2^{p+1} \ell+2^{p}\right)\right) .
$$

Then we have $v_{f^{\prime}}\left(2^{p} \ell-2^{p} i+2^{p-1}\right)=v_{f^{\prime}}\left(2^{p} \ell+2^{p} j-2^{p-1}\right)$. By induction hypothesis, there exists $0 \neq h \in B_{2^{p+1} \ell}$ on variables $x_{2^{p+1}+1}, x_{2^{p+1}+2}, \ldots, x_{2^{p+1}(\ell+1)}$ with $\operatorname{deg}(h)<$ $2^{p} \ell$, such that $h f^{\prime}=0$ or $h\left(f^{\prime}+1\right)=0$.

For the case $h f^{\prime}=0$, let $g=h P_{2^{p}}$. Then, we claim that $f g=0$. To prove the foregoing, we study the weight supports of $g$ and $f$. First, by WS $\left(P_{2^{p}}\right)=\left\{2^{p}\right\}$ and the fact that $P_{2^{p}}$ and $h$ deal with different variables, we know that $W S(g)=$ $\left\{i+2^{p} \mid i \in \mathrm{WS}(h)\right\}$ and $\mathrm{WS}(g) \cap\left\{i \mid 0 \leq i<2^{p}, n-2^{p}<\right.$ $i \leq n\}=\emptyset$. Second, we know that $\mathrm{WS}(f)=\left\{i+2^{p} \mid i \in\right.$ $\left.\mathrm{WS}\left(f^{\prime}\right)\right\} \cup\left\{i \mid 0 \leq i<2^{p}, n-2^{p}<i \leq n, v_{f}(i)=1\right\}$ by the definition of $f^{\prime}$. Third, we have $\mathrm{WS}\left(f^{\prime}\right) \cap \mathrm{WS}(h)=\emptyset$ because $f^{\prime} h=0$. Thus, we have $\mathrm{WS}(f) \cap \mathrm{WS}(g)=\{i+$ $\left.2^{p} \mid i \in \mathrm{WS}\left(f^{\prime}\right)\right\} \cap\left\{i+2^{p} \mid i \in \mathrm{WS}(h)\right\}=\emptyset$, which means $f g=0$. For the case $h\left(f^{\prime}+1\right)=0$, we can prove similarly that $(f+1) g=0$. This contradicts $A I(f)=k$ because $\operatorname{deg}(g)<k$. Therefore,

$$
v_{f}\left(2^{p}(\ell+1)-2^{p} i+2^{p-1}\right)=v_{f}\left(2^{p}(\ell+1)+2^{p} j-2^{p-1}\right)+1
$$

for any $1 \leq i, j \leq \ell$.

Step 2 Assume to the contrary that $v_{f}\left(2^{p-1}\right)=v_{f}\left(2^{p}(\ell+\right.$ 1) $+2^{p} j-2^{p-1}$ ) for $1 \leq j \leq \ell$. To deduce a contradiction, we construct an annihilator $g$ of $f$ or $f+1$ as follows.

Define $g \in \mathrm{SB}_{2^{p+1}(\ell+1)}$ by

$$
\lambda_{g}(\psi)=\left\{\begin{array}{l}
0, \text { if } \psi \geq k \text { or } 2^{p-1} \npreceq \psi, \\
1, \text { if } \psi<k \text { and } 2^{p-1} \preceq \psi .
\end{array}\right.
$$

We claim that $g$ is an annihilator of $f$ or $f+1$. To prove the claim, we study the weight support of $g$. Let $\omega \in \mathrm{WS}(g)$, then $v_{g}(\omega)=1$. By Lemma 2.1, we have

$$
v_{g}(\omega)=\sum_{\psi \preceq \omega} \lambda_{g}(\psi)=\sum_{2^{p-1} \preceq \psi \preceq \omega,} 1
$$

Let

$$
S_{\omega}=\left\{\psi \mid 2^{p-1} \preceq \psi \preceq \omega, \psi<k=2^{p}(l+1)\right\},
$$

then $v_{g}(\omega)=\left|S_{\omega}\right| \bmod 2$, which means $v_{g}(\omega)=1$ if and only if $\left|S_{\omega}\right|$ is odd. Let $\omega=\left(\omega_{m} \omega_{m-1} \cdots \omega_{0}\right)_{2}$ be the binary expansion of $\omega$.

i) If $\omega<k$, we claim that $\left|S_{\omega}\right|$ is odd if and only if $\omega=$ $2^{p-1}$. For $2^{p-1} \npreceq \omega$, there is no $\psi$ satisfying $2^{p-1} \preceq \psi \preceq$ $\omega$, which means $S_{\omega}=\emptyset$. For $2^{p-1} \preceq \omega$, the number of $\psi$ such that $2^{p-1} \preceq \psi \preceq \omega$ is $2^{\operatorname{wt}(\omega)-\operatorname{wt}\left(2^{p-1}\right)}=2^{\operatorname{wt}(\omega)-1}$, which is odd only when $\omega=2^{p-1}$.

ii) If $\omega \geq k$, we claim that $\left|S_{\omega}\right|$ is odd only if $\omega_{p-1}=1$ and $\omega_{t}=0$ for all $0 \leq t<p-1$. Otherwise, if $\omega_{p-1}=0$, then $2^{p-1} \npreceq \omega$ and there is no $\psi$, such that $2^{p-1} \preceq \psi \preceq \omega$, which implies that $S_{\omega}=\emptyset$. If $\omega_{t_{0}}=1$ for some $0 \leq t_{0}<$ $p-1$, and $\psi=\left(\psi_{m} \psi_{m-1} \cdots \psi_{0}\right)_{2}$ is an element of $S_{\omega}$, then it is clear that $\psi^{\prime}=\left(\psi_{m} \cdots \psi_{t_{0}+1} \overline{\psi_{t_{0}}} \psi_{t_{0}-1} \cdots \psi_{0}\right)_{2}$ also satisfies $2^{p-1} \preceq \psi^{\prime} \preceq \omega$ and $\psi^{\prime}<k=2^{p}(\ell+1)$, where $\overline{\psi_{t_{0}}}=\psi_{t_{0}}+1$. Thus, $\psi^{\prime}$ is also an element of $S_{\omega}$, which means the elements in $S_{\omega}$ come into pairs. Thus, $\left|S_{\omega}\right|$ is even, which is a contradiction. Therefore, if $v_{g}(\omega)=1$, then $\omega_{p-1}=1$ and $\omega_{t}=0$ for all $0 \leq t<$ $p-1$.

Combining the results of i) and ii), we have

$$
\mathrm{WS}(g) \subseteq\left\{2^{p-1}, 2^{p}(\ell+1)+2^{p} j-2^{p-1}, 1 \leq j \leq \ell+1\right\} .
$$

Note that $v_{f}\left(2^{p-1}\right)=v_{f}\left(2^{p}(\ell+1)+2^{p} j-2^{p-1}\right), 1 \leq j \leq \ell$, if we can prove that $2^{p}(\ell+1)+2^{p}(\ell+1)-2^{p-1}=n-2^{p-1}$ is not in $\operatorname{WS}(g)$, then we have $f g=0$ or $(1+f) g=0$ since $f$ is constant on the support of $g$. Since $\operatorname{deg}(g)<k$, we have $A I(f)<k$. It is a contradiction, and will end the proof of this part. Therefore, we will prove $v_{g}\left(n-2^{p-1}\right)=0$. Note that once it is proved, we finish the proof of this part.

Let $\omega=n-2^{p-1}=2^{p+1} \ell+2^{p}+2^{p-1}, \psi$ be an element of $S_{\omega}$. According to the definition of $S_{\omega}$, we can see that there exists some integer $0 \leq s \leq \ell$, such that $\psi=2^{p} s+2^{p-1}$. Let $T_{\ell}=\{s \mid s \preceq 2 \ell+1,0 \leq s \leq \ell\}$. Hence we have $\left|S_{\omega}\right|=\left|T_{\ell}\right|$ by the definition of $S_{\omega}$. What we need is to prove that $\left|T_{\ell}\right|$ is even for all $\ell \geq 1$. It is not a difficult task, and the reader can give a proof by himself/herself, or follow the proof below.

If $\ell=2^{r}-1$ for some positive integer $r$, then $2 \ell+1=$ $2^{r+1}-1$. Thus, $s \preceq 2 \ell+1$ for every $0 \leq s \leq \ell$, which means $\left|T_{\ell}\right|=\ell+1=2^{r}$. It is in contradiction with that $\left|S_{\omega}\right|=\left|T_{\ell}\right|$ is odd. Otherwise, let $\ell=\left(\ell_{m} \ell_{m-1} \cdots \ell_{0}\right)_{2}$ be the binary expansion of $\ell$, then there exists some integer $1 \leq$ $t \leq m$ such that $\ell_{m}=\ell_{m-1}=\cdots=\ell_{m-(t-1)}=1$ and $\ell_{m-t}=0$, namely $\ell=(\underbrace{11 \cdots 1}_{t} 0 \ell_{m-t-1} \cdots \ell_{0})_{2}$. Then $2 \ell+$ $1=(\underbrace{11 \cdots 1}_{t} 0 \ell_{m-t-1} \cdots \ell_{0} 1)_{2}$. Let $s=\left(s_{m} s_{m-1} \cdots s_{0}\right)_{2}$, then $s \preceq \stackrel{t}{2} \ell+1$ implies $s_{m-t+1}=0$, which means $s<\ell$ by the structure of $\ell$. Thus, by the definition of $T_{\ell}, s \in T_{\ell}$ if and only if $s \preceq 2 \ell+1$. Since $s<2^{m+1}$ and $(2 \ell+1)_{m+1}=1$, where $(2 \ell+1)_{m+1}$ denotes the $(m+1)^{\text {th }}$ bit in its binary expansion, we have $\left|T_{\ell}\right|=2^{\mathrm{wt}(2 \ell+1)-1}$. Since $\ell \geq 1$, we have $\operatorname{wt}(2 \ell+1)-1>0$ which means $\left|S_{\omega}\right|=\left|T_{\ell}\right|$ also even. Thus, we finish the proof of this step, i.e.,

$$
v_{f}\left(2^{p-1}\right)=v_{f}\left(2^{p}(\ell+1)+2^{p} j-2^{p-1}\right)+1
$$


for $1 \leq j \leq \ell$.

Step 3 Assume to the contrary that $v_{f}\left(2^{p}(\ell+1)-2^{p} i+\right.$ $\left.2^{p-1}\right)=v_{f}\left(2^{p+1}(\ell+1)-2^{p-1}\right)$, for $1 \leq i \leq \ell$. Similar with step 2 , by using $g^{\prime}$ instead of $g$, where $g^{\prime}\left(x_{1}, \ldots, x_{n}\right)=$ $g\left(x_{1}+1, \ldots, x_{n}+1\right)$, we can get $f g^{\prime}=0$ or $(f+1) g^{\prime}=0$, which contradicts $\mathrm{AI}(f)=k$. Thus,

$$
v_{f}\left(2^{p}(\ell+1)-2^{p} i+2^{p-1}\right)=v_{f}\left(2^{p+1}(\ell+1)-2^{p-1}\right)+1
$$

for any $1 \leq i \leq \ell$.

Step 4 Combining the above three steps, we have

$$
\begin{array}{rlr} 
& v_{f}\left(2^{p-1}\right) & \\
= & v_{f}\left(2^{p}(\ell+1)+2^{p} j-2^{p-1}\right)+1 & \text { by (3) } \\
= & v_{f}\left(2^{p}(\ell+1)-2^{p} i+2^{p-1}\right) & \text { by (2) } \\
= & v_{f}\left(2^{p+1}(\ell+1)-2^{p-1}\right)+1 & \text { by (4) }
\end{array}
$$

for any $1 \leq i, j \leq \ell$. Thus, $v_{f}\left(2^{p}(\ell+1)-2^{p} i+2^{p-1}\right)=$ $v_{f}\left(2^{p}(\ell+1)+2^{p} j-2^{p-1}\right)+1$ for $i=j=\ell+1$.

Combining the above four steps, $v_{f}\left(2^{p}(\ell+1)-2^{p} i+\right.$ $\left.2^{p-1}\right)=v_{f}\left(2^{p}(\ell+1)+2^{p} j-2^{p-1}\right)+1$ holds for any $1 \leq i, j \leq \ell+1$. Therefore, the theorem is also true for $\mu=\ell+1$. This completes the proof.

In Lemma 3.1, $n$ should be a multiple of 4 . The following theorem generalizes Lemma 3.1 to a wider situation, where $n$ can be any even number.

Theorem 3.1: Let $n=2^{p+1} \mu+2 m$ with $p, \mu \geq 1$ and $0 \leq m<2^{p}, f \in \mathrm{SB}_{n}$. If $\mathrm{AI}(f)=k$, then

$v_{f}\left(2^{p} \mu+m-2^{p} i+2^{p-1}\right)=v_{f}\left(2^{p} \mu+m+2^{p} j-2^{p-1}\right)+1$

for any $1 \leq i, j \leq \mu$.

Proof: Assume to the contrary that $v_{f}\left(2^{p} \mu+m-2^{p} i+\right.$ $\left.2^{p-1}\right)=v_{f}\left(2^{p} \mu+m+2^{p} j-2^{p-1}\right)$ for some $1 \leq i, j \leq \mu$. Let $f^{\prime} \in \mathrm{SB}_{2^{p+1} \mu}$ on variables $x_{2 m+1}, x_{2 m+2}, \ldots, x_{2^{p+1} \mu+2 m}$, be defined as

$$
v_{f^{\prime}}=\left(v_{f}(m), v_{f}(m+1), \ldots, v_{f}\left(2^{p+1} \mu+m\right)\right) .
$$

Then we have $v_{f^{\prime}}\left(2^{p} \mu-2^{p} i+2^{p-1}\right)=v_{f^{\prime}}\left(2^{p} \mu+2^{p} j-2^{p-1}\right)$. By Lemma 3.1 $\mathrm{AI}\left(f^{\prime}\right)<2^{p} \mu$, thus there exists $0 \neq h \in$ $B_{2^{p+1} \mu}$ with $\operatorname{deg}(h)<2^{p} \mu$ such that $f^{\prime} h=0$ or $\left(f^{\prime}+1\right) h=0$. Let $g=h P_{m}$. Following the argument of step 1 in Lemma 3.1 we claim that $f g=0$ or $(f+1) g=0$ with $\operatorname{deg}(g)<2^{p} \mu+$ $m=k$, which contradicts $\operatorname{AI}(f)=k$. Therefore, $v_{f}\left(2^{p} \mu+\right.$ $\left.m-2^{p} i+2^{p-1}\right)=v_{f}\left(2^{p} \mu+m+2^{p} j-2^{p-1}\right)+1$ for any $1 \leq i, j \leq \mu$.

For example, when $n=2 k=14$, we have

- if $p=1, \mu=3, m=1$, then $\left\{2^{p} \mu+m-2^{p} i+2^{p-1}, 2^{p} \mu+\right.$ $\left.m+2^{p} j-2^{p-1} \mid 1 \leq i, j \leq \mu\right\}=\{2,4,6,8,10,12\}$,

- if $p=2, \mu=1, m=3$, then $\left\{2^{p} \mu+m-2^{p} i+2^{p-1}, 2^{p} \mu+\right.$ $\left.m+2^{p} j-2^{p-1} \mid 1 \leq i, j \leq \mu\right\}=\{5,9\}$.

Theorem 3.1 sets constraints on $v_{f}(\omega)$ for $\omega \in\{2,4,5,6,8,9$, $10,12\}$.

For convenience of description, we introduce a partial order on nonnegative integers denoted as $\preceq^{\prime}$.
Definition 3.1: Given two binary expansions of nonnegative integers $a=\left(a_{s}, a_{s-1}, \ldots, a_{0}\right)_{2}, b=\left(b_{\ell}, b_{\ell-1}, \ldots, b_{0}\right)_{2}, 1 \leq$ $\ell \leq s$, we define

$$
\begin{aligned}
& b \preceq^{\prime} a \Leftrightarrow b=0 \text { or } b_{i}=a_{i} \text { for all } 0 \leq i \leq \ell ; \\
& b \prec^{\prime} a \Leftrightarrow b \preceq^{\prime} a \text { and } b \neq a .
\end{aligned}
$$

For example, we have $3 \prec^{\prime} 7$ because $7=(111)_{2}$ and $3=$ $(11)_{2}$.

For any nonnegative integer $k$, let $\mathrm{B}^{k}=\left\{i, 2 k-i \mid i \prec^{\prime} k\right\}$. By the definition of $\prec^{\prime},\left|\mathrm{B}^{k}\right|=2 \mathrm{wt}(k)$.

Definition 3.2: For any positive integer $n=2 k$, we divide the set $\{0,1, \ldots, n\}$ into a series of subsets $\mathrm{A}_{i}^{k}$, where

$$
\begin{aligned}
& \mathrm{A}_{0}^{k}=\{k\}, \\
& \mathrm{A}_{i}^{k}=\left\{k-(2 j+1) 2^{i-1}, k+(2 j+1) 2^{i-1} \mid 0 \leq j \leq\right. \\
& \left.\frac{\left\lfloor\frac{k}{2^{i}-1}\right\rfloor-1}{2}\right\},
\end{aligned}
$$

for $1 \leq i \leq\left\lfloor\log _{2} n\right\rfloor$. The superscript $k$ may be omitted if there is no confusion.

The union of sets $\left\{(2 j+1) 2^{i-1} \mid j \in N\right\}$ over all $i \in N^{+}$is a partition of $N^{+}$, so these subsets have no intersection with each other, and $\{0,1, \ldots, n\}=\bigcup_{i=0}^{\left\lfloor\log _{2} n\right\rfloor} \mathrm{A}_{i}$.

For example, when $n=2 k=14$, we have $A_{0}^{7}=\{7\}, A_{1}^{7}=$ $\{0,2,4,6,8,10,12,14\}, \mathrm{A}_{2}^{7}=\{1,5,9,13\}, \mathrm{A}_{3}^{7}=\{3,11\}$.

The main intuition of sets $A_{i}^{k}$ and $\prec^{\prime}$ could be explained by the binary expansion of $k$, where $k=\left(k_{m}, \ldots, k_{0}\right)_{2}$ with $k_{m} \neq 0$. For any $a \prec^{\prime} k$, it is easy to verify that $a=\left(\overline{k_{j}}, k_{j-1}, \ldots, k_{0}\right)_{2}$, where $k_{j}=1$ and $\overline{k_{j}}=k_{j}+1=0$. And for every $\omega \in A_{i}^{k}$, the binary expansion of $\omega$ is $\left(*, \overline{k_{i-1}}, k_{i-2}, \ldots, k_{0}\right)_{2}$, where $*$ is an arbitrary binary string, which means the right-most $i$ bits of the binary expansion of $\omega$ are exactly $\overline{k_{i-1}}, k_{i-2}, \ldots, k_{0}$.

The following Lemma contains some properties of $A_{i}^{k}$, as well as the partial order $\prec^{\prime}$.

Lemma 3.2: Supposing $k=\left(k_{m}, \ldots, k_{1}, k_{0}\right)_{2}$, by the definition of $\mathrm{A}_{i}^{k}$, a simple calculation gives the following:

1) if $j \in \mathrm{A}_{i}^{k}$, then $2 k-j \in \mathrm{A}_{i}^{k}$;

2) for any $0 \leq j \leq 2 k, j=\left(j_{m}, \ldots, j_{1}, j_{0}\right)_{2}, j \in \mathrm{A}_{i}^{k}$ if and only if $\left(j_{i-1}, j_{i-2}, \ldots, j_{1}, j_{0}\right)=\left(\overline{k_{i-1}}, k_{i-2}, \ldots, k_{1}, k_{0}\right)$, in particular, $\mathrm{A}_{0}^{k}=\{k\}, \mathrm{A}_{\left\lfloor\log _{2} k\right\rfloor+1}^{k}=\left\{k-2^{\left\lfloor\log _{2} k\right\rfloor}, k+2^{\left\lfloor\log _{2} k\right\rfloor}\right\}$;

3) $\mathrm{A}_{i}^{k}$ contains an element $\left(\overline{k_{i-1}}, k_{i-2}, \ldots, k_{1}, k_{0}\right)_{2} \prec^{\prime} k$ in $B^{k}$ if and only if $k_{i-1}=1$.

We explain the reason why we define the sets $\mathrm{A}_{i}^{k}$ and $\mathrm{B}^{k}$. Given $n=2 k=2^{p+1} \mu+2 m\left(p, \mu \geq 1,0 \leq m<2^{p}\right)$, it is easy to verify that

$\mathrm{A}_{p}^{k} \supseteq\left\{2^{p} \mu+m-2^{p} i+2^{p-1}, 2^{p} \mu+m+2^{p} j-2^{p-1} \mid 1 \leq i, j \leq \mu\right\}$,

which means the $\omega$ of $v_{f}(\omega)$ from the same equation defined by Theorem 3.1 are all included in the same $\mathrm{A}_{p}^{k}$. But $\mathrm{A}_{p}^{k}$ may contain two extra elements, which are

$$
\begin{aligned}
\mathrm{A}_{p}^{k}- & \left\{2^{p} \mu+m-2^{p} i+2^{p-1}, 2^{p} \mu+m+2^{p} j-2^{p-1} \mid 1 \leq i, j \leq \mu\right\} \\
& = \begin{cases}\left\{m-2^{p-1}, n-m+2^{p-1}\right\}, & m-2^{p-1} \geq 0, \\
\emptyset, & m-2^{p-1}<0 .\end{cases}
\end{aligned}
$$


For the case $m-2^{p-1} \geq 0$, since $m<2^{p}$, we have $m=$ $2^{p-1}+s\left(0 \leq s<2^{p-1}\right)$, which means $m-2^{p-1} \prec^{\prime} m \prec^{\prime} k$. Besides, by the definition of partial order $\prec^{\prime}$, if $m+2^{p} \mu=k$ $\left(m<2^{p}, \mu>1\right)$, then $2^{p} \mu+m-2^{p} i+2^{p-1} \npreceq^{\prime} k$ for $1 \leq i \leq \mu$. Thus, Theorem 3.1 shows constraints on $v_{f}(\omega)$ if and only if $\omega \swarrow^{\prime} k$, namely $\omega \in\{0,1, \ldots, n\}-\mathrm{B}^{k}-\{k\}$.

Equipped with these notations and basic properties, we can restate Theorem 3.1 concisely.

Corollary 3.1: Let $n=2 k$ and $f \in \mathrm{SB}_{n}$. If $\mathrm{AI}(f)=k$, then for any $1 \leq p \leq\left\lfloor\log _{2} k\right\rfloor$ and $i, j \in \mathrm{A}_{p}^{k}-\mathrm{B}^{k}-\{k\}$ with $i \leq j<k$, we have $v_{f}(i)=v_{f}(j)=v_{f}(n-j)+1=v_{f}(n-i)+1$.

Corollary 3.1 shows the constraints related to the values of $v_{f}$ on $\{0,1, \ldots, n\}-\mathrm{B}^{k}-\{k\}$. In what follows, we will discuss $v_{f}$ on $B^{k} \cup\{k\}$.

Theorem 3.2: Let $f \in \mathrm{SB}_{n}$. For any $t \prec^{\prime} k, t \neq k-$ $2^{\left\lfloor\log _{2} k\right\rfloor}$, assume $t \in \mathrm{A}_{p}$. If

$$
\begin{aligned}
v_{f}(t)+1 & =v_{f}\left(t+2^{p}\right)=\cdots=v_{f}\left(k-2^{p-1}\right) \\
& =v_{f}\left(k+2^{p-1}\right)+1=\cdots=v_{f}\left(n-t-2^{p}\right)+1 \\
& =v_{f}(n-t),
\end{aligned}
$$

then $\operatorname{AI}(f)<k$.

Proof: Notice that $\mathrm{A}_{p}=\left\{t, t+2^{p}, \ldots, k-2^{p-1}, k+2^{p-1}\right.$, $\left.\ldots, n-t-2^{p-1}, n-t\right\}$. If $k=2^{q}$ for some $q$, then only one $i(i=0)$ satisfies $i \prec^{\prime} k$ and $0=k-2^{\left\lfloor\log _{2} k\right\rfloor}$. It contradicts the conditions in this theorem. Therefore, we have $k \neq 2^{q}$ for any integer $q$. We only need to consider $\operatorname{wt}\left(v_{f}\right)=k$ or $k+1$. Otherwise, we have $A I(f)<k$ by Lemma 2.5. Without loss of generality, we assume $\operatorname{wt}\left(v_{f}\right)=k$. Otherwise when $\operatorname{wt}\left(v_{f}\right)=k+1$, we can replace $f$ by $f+1$ instead.

We will prove that there exists a nonzero symmetric Boolean function $g$ with degree less than $k$, such that $f g=0$ which implies $A I(f)<0$. Let $g=\sum_{i=0}^{k-1} \lambda_{g}(i) \sigma_{i}$. Notice that $f g=$ 0 if and only if for every $v_{f}(i)=1$ we have

$$
v_{g}(i)=\sum_{\substack{j \prec i \\ 0 \leq j \leq k-1}} \lambda_{g}(j)=0
$$

by Lemma 2.1. Then, we can get a system of homogeneous linear equations on variables $\lambda_{g}(0), \ldots, \lambda_{g}(k-1)$ with $w t\left(v_{f}\right)=k$ equations. The number of equations and unknowns of the equation system are both $k$. In what follows, we will show that there are two same equations. Thus there must exist a nonzero solution of $\lambda_{g}(0), \ldots, \lambda_{g}(k-1)$, which implies the existence of $g$.

Since $k \neq 2^{q}$ for any integer $q$, we assume $2^{\ell-1}<k<2^{\ell}$. Thus, we have $t<2^{\ell-1}$ and $\left\lfloor\log _{2} k\right\rfloor=\ell-1$. Since $t \neq$ $k-2^{\left.\log _{2} k\right\rfloor}$, we have $2 t \neq n-2^{\ell} \Rightarrow n-t \neq t+2^{\ell} \Rightarrow$ $n-t-2^{\ell} \neq t$. According to the definition of $A_{p}$, we have $t+2^{\ell}, n-t-2^{\ell} \in \mathrm{A}_{p}$.

For the case $v_{f}(t)=1$, since $t+2^{\ell} \in A_{p}, t+2^{\ell}>k$ and $n-t \neq t+2^{\ell}$, we have $v_{f}\left(t+2^{\ell}\right)=v_{f}(t)=1$ by $[5$. Consider the equations

$$
v_{g}(t)=\sum_{\substack{i \preceq t \\ 0 \leq i<k}} \lambda_{g}(i)=0
$$

and

$$
v_{g}\left(t+2^{\ell}\right)=\sum_{\substack{i \preceq t+2^{\ell} \\ 0 \leq i<k}} \lambda_{g}(i)=0 .
$$

It is easy to see that $i \preceq t$ is equivalent to $i \preceq t+2^{\ell}$ for $0 \leq i<k$; thus, the two equations above are exactly the same.

For the case $v_{f}(t)=0$, we could prove $v_{f}(n-t)=v_{f}(n-$ $\left.t-2^{\ell}\right)=1$ similar to the case $v_{f}(t)=1$. It is similar to verify that equations $v_{g}(n-t)=0$ and $v_{g}\left(n-t-2^{\ell}\right)=0$ are exactly the same.

Therefore, the nonzero symmetric annihilator with degree less than $k$ always exists, and $\mathrm{AI}(f)<k$.

For a given $k$, the values $t$ and $n-t$ such that $t \prec^{\prime} k$ and $t \neq k-2^{\left\lfloor\log _{2} k\right\rfloor}$ can occur in Theorem 3.2, but are excluded in Corollary 3.1. Theorem 3.2 focuses on the relationship between $v_{f}(\omega)$ where $\omega \in\{t, n-t\}$, and $v_{f}(\omega)$ where $\omega \in A_{p}-\{t, n-t\}$. In the following theorem, we will consider $v_{f}\left(k-2^{\left\lfloor\log _{2} k\right\rfloor}\right)$ and $v_{f}\left(n-k+2^{\left\lfloor\log _{2} k\right\rfloor}\right)$, which are excluded in Theorem 3.2.

Theorem 3.3: Let $n=2 k$ and $f \in \mathrm{SB}_{n}$. If $A I(f)=k$, then there does not exist more than one integer $i$, such that $i \prec^{\prime} k$ and $v_{f}(i)=v_{f}(n-i)$.

Proof: When $k=2^{q}$ and $q$ is an integer, there is only one $i(i=0)$ satisfying $i \prec^{\prime} k$. The conclusion is trivial. Therefore, we only need to consider the case $k \neq 2^{q}$ for any integer $q$. By Lemma2.5, we only need to consider wt $\left(v_{f}\right)=k$ or $k+1$. Without loss of generality, we assume $\operatorname{wt}\left(v_{f}\right)=k$. Otherwise, when $\operatorname{wt}\left(v_{f}\right)=k+1$, we can replace $f$ by $f+1$ instead.

Assume to the contrary that there exist more than one $i$ such that $i \prec^{\prime} k$ and $v_{f}(i)=v_{f}(n-i)$. We will show the existence of a nonzero symmetric Boolean function $g$ with degree less than $k$ such that $f g=0$, which is contradicted with $A I(f)=k$.

Let $g=\sum_{i=0}^{k-1} \lambda_{g}(i) \sigma_{i}$. Notice that $f g=0$ if and only if for every $v_{f}(i)=1$ we have

$$
v_{g}(i)=\sum_{\substack{j \preceq i \\ 0 \leq j \leq k-1}} \lambda_{g}(j)=0
$$

by Lemma 2.1. Then, we can get a system of homogeneous linear equations on variables $\lambda_{g}(0), \ldots, \lambda_{g}(k-1)$ with $w t\left(v_{f}\right)=k$ equations. Notice the number of equations and unknowns are both $k$. In what follows, we will show that there are two same equations; thus, there must exist a nonzero solution of $\lambda_{g}(0), \ldots, \lambda_{g}(k-1)$, which implies the existence of $g$.

We claim that there exists at least one $i_{1}$ such that $i_{1} \prec^{\prime} k$ and $v_{f}\left(i_{1}\right)=v_{f}\left(n-i_{1}\right)=1$ under our assumption that more than one $i \prec^{\prime} k$ exist s.t. $v_{f}(i)=v_{f}(n-i)$. Else, suppose $v_{f}(i)=v_{f}(n-i)=0$ for all $i \prec^{\prime} k$ and $v_{f}(i)=v_{f}(n-i)$. It is easy to verify that $\operatorname{wt}(f)<k$, because $v_{f}(i)=v_{f}(n-$ $i)+1$ for other $i \prec^{\prime} k$ and $v_{f}(\psi)=v_{f}(n-\psi)+1$ for all $\psi \in\{0,1, \ldots, n\}-B^{k}-\{k\}$ due to Corollary 3.1. This is contradicted with wt $(f)=k$. Thus the existence of $i_{1}$ is guaranteed. Since $k \neq 2^{q}$ for any $q$, we assume $2^{\ell-1}<k<$ $2^{\ell}$. 
Case 1: If $i_{1} \neq k-2^{\left\lfloor\log _{2} k\right\rfloor}$, assume $i_{1} \in A_{p}^{k}$. Since $A I(f)=k$, according to Corollary 3.1, we have

$$
\begin{aligned}
v_{f}\left(i_{1}\right) & =v_{f}\left(i_{1}+2^{p}\right)=\cdots=v_{f}\left(k-2^{p-1}\right) \\
& =v_{f}\left(k+2^{p-1}\right)+1=\cdots=v_{f}\left(n-i_{1}-2^{p}\right)+1 \\
& =v_{f}\left(n-i_{1}\right)=1,
\end{aligned}
$$

or

$$
\begin{aligned}
v_{f}\left(i_{1}\right) & =v_{f}\left(i_{1}+2^{p}\right)+1=\cdots=v_{f}\left(k-2^{p-1}\right)+1 \\
& =v_{f}\left(k+2^{p-1}\right)=\cdots=v_{f}\left(n-i_{1}-2^{p}\right) \\
& =v_{f}\left(n-i_{1}\right)=1 .
\end{aligned}
$$

By the definition of $A_{p}^{k}$, we have $i_{1}+2^{\ell}, n-i_{1}-2^{\ell} \in A_{p}^{k}$. Since $i_{1}+2^{\ell}>k$ and $n-i_{1}-2^{\ell}<k$, we have $v_{f}\left(n-i_{1}\right)=$ $v_{f}\left(n-i_{1}-2^{\ell}\right)=1$ for (6) and $v_{f}\left(i_{1}\right)=v_{f}\left(i_{1}+2^{\ell}\right)=1$ for (7). Then, similar with the proof in Theorem 3.2, we can prove that they are two same equations in both cases, i.e., $v_{g}\left(n-i_{1}\right)=0$ is equivalent to $v_{g}\left(n-i_{1}-2^{\ell}\right)=0$ and $v_{g}\left(i_{1}\right)=0$ equivalent to $v_{g}\left(i_{1}+2^{\ell}\right)=0$.

Case 2: For $i_{1}=k-2^{\left\lfloor\log _{2} k\right\rfloor}$, we have $i_{1}=k-2^{\ell-1} \Rightarrow$ $2 i_{1}=n-2^{\ell} \Rightarrow n-i_{1}=2^{\ell}+i_{1}$. Thus, we have $v_{f}\left(i_{1}\right)=$ $v_{f}\left(n-i_{1}\right)=v_{f}\left(i_{1}+2^{\ell}\right)=1$. Consider the following two equations,

$$
\begin{aligned}
v_{g}\left(n-i_{1}\right) & =v_{g}\left(2^{\ell}+i_{1}\right) \\
& =\sum_{\substack{i \preceq 2^{\ell}+i_{1} \\
0 \leq i<k}} \lambda_{g}(i)=0,
\end{aligned}
$$

and

$$
v_{g}\left(i_{1}\right)=\sum_{\substack{i \preceq i_{1} \\ 0 \leq i<k}} \lambda_{g}(i)=0 .
$$

For $0 \leq i<k$, we have $i \preceq 2^{\ell}+i_{1}$ if and only if $i \preceq i_{1}$. Thus, the above two equations are equivalent.

Therefore, the nonzero symmetric annihilator with degree less than $k$ always exists, which is contradictory to $A I(f)=k$. Thus, there cannot exist more than one integer $i$, such that $i \prec^{\prime} k$ and $v_{f}(i)=v_{f}(n-i)$.

For the case one $t$ exists such that $t \prec^{\prime} k$ and $v_{f}(t)=$ $v_{f}(n-t)$, there exists another constraint, namely Theorem 3.4. This theorem is the last necessary condition for even-variable symmetric Boolean functions to reach maximum algebraic immunity, which considers all the triples $\left(v_{f}(t), v_{f}(k), v_{f}(n-t)\right)$ when $t \prec^{\prime} k$.

Theorem 3.4: Let $n=2 k, f \in \mathrm{SB}_{n}$. If $\mathrm{AI}(f)=k$, then for any $t \prec^{\prime} k,\left(v_{f}(t), v_{f}(k), v_{f}(n-t)\right) \notin\{(0,0,0),(1,1,1)\}$.

Proof: According to Corollary 3.1, for any $i$ and any $p$ such that $i \in A_{p}-B^{k}-\{k\}$, we have $v_{f}(i)=v_{f}(n-i)+1$. By Theorem 3.3, for all elements of $\mathrm{B}^{k}$, at most one $t$ could exist such that $t \prec^{\prime} k$ and $v_{f}(t)=v_{f}(n-t)$. Therefore, $\operatorname{wt}\left(v_{f}\right)=$ $k-1$ if $\left(v_{f}(t), v_{f}(k), v_{f}(n-t)\right)=(0,0,0)$ for some $t \prec^{\prime} k$ and $\operatorname{wt}\left(v_{f}\right)=k+2$ if $\left(v_{f}(t), v_{f}(k), v_{f}(n-t)\right)=(1,1,1)$ for some $t \prec^{\prime} k$. By Lemma 2.5, we know either case is impossible.

In the end of this section, we take out all even-variable symmetric Boolean functions satisfying all the necessary conditions to achieve maximum algebraic immunity into the following two classes.

Definition 3.3: Define two classes of symmetric Boolean functions on $n$ variable, $n=2 k$, as follows.

Class 1: For any $\mathrm{A}_{p}, 1 \leq p \leq\left\lfloor\log _{2} n\right\rfloor$, and $i, j \in \mathrm{A}_{p}$,

$$
v_{f}(i)= \begin{cases}v_{f}(j)+1, & \text { if } i<k<j \text { or } j<k<i, \\ v_{f}(j), & \text { otherwise. }\end{cases}
$$

Class 2: There is a function $g$ contained in Class 1 and an integer $t \prec^{\prime} k$ such that

$$
\begin{aligned}
& v_{f}=v_{g}+e_{t}+\delta e_{k}, \text { or } \\
& v_{f}=v_{g}+e_{n-t}+\delta^{\prime} e_{k},
\end{aligned}
$$

where

$$
\delta=v_{g}(t)+v_{g}(k),
$$

and

$$
\delta^{\prime}=v_{g}(n-t)+v_{g}(k)
$$

If there is no $t$ such that $t \prec^{\prime} k$ and $v_{f}(t)=v_{f}(n-t)$, then $f$ is contained in Class 1. If such $t$ exists, $f$ is contained in Class 2. Class 2 is defined based on Class 1 .

Theorem 3.5: Suppose $f \in \mathrm{SB}_{n}, n=2 k$. If $\mathrm{AI}(f)=k$, then $f$ is in Class 1 or 2 .

Proof: If for any $t \prec^{\prime} k, v_{f}(t) \neq v_{f}(n-t)$, we will prove $f$ is in Class 1. By Theorem 3.1 and Theorem 3.2 we know that $v_{f}(i)=v_{f}(j)+1$ for all $i, j \in A_{p}^{k}$ and $i<k<j$, which satisfies the definition of Class 1 functions.

If there is some $t \prec^{\prime} k, v_{f}(t)=v_{f}(n-t)$. By Theorem 3.3. we know at most one such $t$ can exist.

When $v_{f}(t)=v_{f}(n-t)=0$, by Theorem 3.4 we know $v_{f}(k)=1$. Let $v_{g_{1}}=v_{f}+e_{t}$ and $v_{g_{2}}=v_{f}+e_{n-t}$. By Theorem 3.1 and Theorem 3.2, $g_{1}, g_{2}$ are in Class 1 and $v_{f}=$ $v_{g}+e_{t}+\delta e_{k}$ and $v_{f}=v_{g}+e_{n-t}+\delta^{\prime} e_{k}$, where $\delta, \delta^{\prime}$ are in Definition 3.3. When $v_{f}(t)=v_{f}(n-t)=1$, the proof is the same.

Classes 1 and 2 consist of all functions satisfying the necessary conditions to reach maximum algebraic immunity. In the following sequel, we will prove that they do reach maximum AI, i.e., the necessary conditions are sufficient.

\section{Functions in Class 1 Have Maximum Algebraic IMMUNITY}

Given a positive integer $k=\left(k_{m}, \ldots, k_{1}, k_{0}\right)_{2}$ and any nonnegative integer $i$, we denote the vector

$$
\left(\varepsilon_{i, 0}, \ldots, \varepsilon_{i, k-2}, \varepsilon_{i, k-1}\right) \in \mathrm{F}_{2}^{k}
$$

by $\varepsilon_{i}^{k}$ or simply $\varepsilon_{i}$ if there is no confusion, where

$$
\varepsilon_{i, j}=\left\{\begin{array}{l}
1, \text { if } j \preceq i, \\
0, \text { otherwise. }
\end{array}\right.
$$


Equivalently,

$$
\varepsilon_{i}=\sum_{\substack{j \preceq i \\ 0 \leq j \leq k-1}} e_{j} .
$$

Furthermore, the inverse representation is easy to obtain, namely,

$$
e_{i}=\sum_{\substack{j \preceq i \\ 0 \leq j \leq k-1}} \varepsilon_{j} .
$$

Therefore, $\left\{\varepsilon_{0}, \varepsilon_{1}, \ldots, \varepsilon_{k-1}\right\}$ is a basis of $\mathrm{F}_{2}^{k}$. Moreover, $\left\{\varepsilon_{k+1}, \varepsilon_{k+2}, \ldots, \varepsilon_{2 k}\right\}$ is also a basis of $\mathrm{F}_{2}^{k}$, as the following lemma states.

Lemma 4.1: $\left\{\varepsilon_{0}, \varepsilon_{1}, \ldots, \varepsilon_{k-1}\right\}$ and $\left\{\varepsilon_{k+1}, \varepsilon_{k+2}, \ldots, \varepsilon_{2 k}\right\}$ are two bases of $\mathrm{F}_{2}^{k}$.

Proof: By (8), we claim $\left\{\varepsilon_{0}, \varepsilon_{1}, \ldots, \varepsilon_{k-1}\right\}$ is a basis of $\mathrm{F}_{2}^{k}$. For $\left\{\varepsilon_{k+1}, \varepsilon_{k+2}, \ldots, \varepsilon_{2 k}\right\}$, let's consider a system of homogeneous equation on variables $x_{0}, x_{1}, \ldots, x_{k-1}$ :

$$
\left(\begin{array}{c}
\varepsilon_{k+1} \\
\varepsilon_{k+2} \\
\vdots \\
\varepsilon_{2 k}
\end{array}\right) X^{\mathrm{T}}=0
$$

where $X=\left(x_{0}, x_{1}, \ldots, x_{k-1}\right) \in \mathrm{F}_{2}^{k}$. We assume that this equation system has a nonzero solution $\lambda=\left(\lambda_{0}, \lambda_{1}, \ldots\right.$, $\left.\lambda_{k-1}\right) \in \mathrm{F}_{2}^{k}$. Let $g(x)=\sum_{i=0}^{k-1} \lambda_{i} \sigma_{i} \in \mathrm{SB}_{n}$, then $\lambda_{g}=$ $\left(\lambda_{0}, \lambda_{1}, \ldots, \lambda_{k-1}, 0,0, \ldots, 0\right) \in \mathrm{F}_{2}^{2 k+1}$. According to the assumption, for $k+1 \leq i \leq 2 k$,

$$
v_{g}(i)=\sum_{j \preceq i} \lambda_{g}(j)=\sum_{\substack{j \preceq i \\ 0 \leq j \leq k}} \lambda_{j}=\varepsilon_{i} \lambda^{T}=0,
$$

which means $v_{g}(i)=0$ holds for $k+1 \leq i \leq 2 k$. Let $f \in \mathrm{SB}_{n}$ be the function in Lemma 2.3. Thus, $f g=0$ and $\operatorname{deg}(g)<k$.

However, by Lemma 2.3, $\operatorname{AI}(f)=k$. Therefore, we have $g=0$, so the above system can have only one solution $X=0$. Thus, $\left\{\varepsilon_{k+1}, \varepsilon_{k+2}, \ldots, \varepsilon_{2 k}\right\}$ is a basis of $\mathrm{F}_{2}^{k}$.

For any $0 \leq i \leq\left\lfloor\log _{2} k\right\rfloor$, let

$$
\begin{gathered}
\mathrm{U}_{i}=\left\{\varepsilon_{j} \mid j \in \mathrm{A}_{i+1}^{k}, 0 \leq j \leq k-1\right\}, \\
\mathrm{V}_{i}=\left\{\varepsilon_{j} \mid j \in \mathrm{A}_{i+1}^{k}, k+1 \leq j \leq 2 k\right\},
\end{gathered}
$$

and

$$
\mathrm{W}_{i} \in\left\{\mathrm{U}_{i}, \mathrm{~V}_{i}\right\} .
$$

Lemma 4.2: $\mathrm{U}_{0}$ or $\mathrm{V}_{0}$, union $\mathrm{U}_{1}$ or $\mathrm{V}_{1}, \ldots$, union $\mathrm{U}_{\left\lfloor\log _{2} k\right\rfloor}$ or $\mathrm{V}_{\left\lfloor\log _{2} k\right\rfloor}$, denoted by $\bigcup_{i=0}^{\left\lfloor\log _{2} k\right\rfloor} \mathrm{W}_{i}$, is a basis of $\mathrm{F}_{2}^{k}$.

Proof: First, we prove that all vectors in $\mathrm{V}_{p}$ can be written as linear combinations of vectors in $\bigcup_{i=0}^{p} \mathrm{U}_{i}$, i.e., $\mathrm{V}_{p} \subseteq \operatorname{span}\left(\bigcup_{i=0}^{p} \mathrm{U}_{i}\right)$. Take an arbitrary vector in $\mathrm{V}_{p}$, denoted by $\varepsilon_{t}$, for some $t \in \mathrm{A}_{p+1}$ and $k+1 \leq t \leq 2 k$. Then, $t=(*$, $\left.\overline{k_{p}}, \ldots, k_{1}, k_{0}\right)_{2}$ by Lemma 3.2, where $*$ is a binary string of arbitrary length. We can expand $\varepsilon_{t}$ as follows:

$$
\begin{aligned}
\varepsilon_{t} & =\sum_{\substack{i \preceq t \\
0 \leq i \leq k-1}} e_{i}=\sum_{\substack{i \preceq t \\
0 \leq i \leq k-1}} \sum_{j \preceq i} \varepsilon_{j} \\
& =\sum_{\substack{j \preceq t \\
0 \leq j \leq k-1}} \varepsilon_{j} \sum_{\substack{j \preceq i \preceq t \\
0 \leq i \leq k-1}} 1 .
\end{aligned}
$$

Therefore, $\varepsilon_{t}$ can be written as a linear combination of vectors in $\bigcup_{i=0}^{\left\lfloor\log _{2} k\right\rfloor} \mathrm{U}_{i}$. For any $\varepsilon_{j} \notin \bigcup_{i=0}^{p} U_{i}$, i.e., $j=$ $\left(*, k_{p}, \ldots, k_{1}, k_{0}\right)_{2}$ and $j \leq k-1$, we calculate the coefficient of $\varepsilon_{j}$, which is

$$
\sum_{\substack{j \preceq i \preceq t \\ 0 \leq i \leq k}} 1=\sum_{\substack{\left(*, k_{p}, \ldots, k_{1}, k_{0}\right)_{2} \preceq i \preceq\left(*, \overline{k_{p}}, \ldots, k_{1}, k_{0}\right)_{2} \\ 0 \leq i \leq k-1}} 1 .
$$

When $k_{p}=1$, there is no $i$ that satisfies the constraints; thus, equation (9) is 0 . When $k_{p}=0$, if there is an $i=\left(*, 0, i_{p-1}, \ldots, i_{2}, i_{1}, i_{0}\right)_{2}$ that satisfies constraints $\left(*, k_{p}, \ldots, k_{1}, k_{0}\right)_{2} \preceq i \preceq\left(*, \overline{k_{p}}, \ldots, k_{1}, k_{0}\right)_{2}$ and $i \leq k-1$, it's not hard to see $i+2^{p}=\left(*, 1, i_{p-1}, \ldots, i_{2}, i_{1}, i_{0}\right)_{2}$ also satisfies the above constraints and vice versa. Therefore, all $1 \mathrm{~s}$ counted in equation (9) are in pairs; thus, equation (9) is 0 . Since all $\varepsilon_{j} \notin \bigcup_{i=0}^{p} \mathrm{U}_{i}$ will not exist in the expansion of $\varepsilon_{t} \in \mathrm{V}_{p}$, we conclude that $\mathrm{V}_{p} \subseteq \operatorname{span}\left(\bigcup_{i=0}^{p} \mathrm{U}_{i}\right)$.

Second, we use math induction to prove that the vector space spanned by $\bigcup_{i=0}^{p} \mathrm{U}_{i}$ is that spanned by $\bigcup_{i=0}^{p} \mathrm{~W}_{i}$, for $p=0,1, \ldots,\left\lfloor\log _{2} k\right\rfloor$. The induction parameter is $p$.

Basis: We claim that $\operatorname{span}\left(\mathrm{U}_{0}\right)=\operatorname{span}\left(\mathrm{V}_{0}\right)$.

By Lemma 4.1, there is no linear dependence in $\mathrm{U}_{0}$ and $\mathrm{V}_{0}$, so $\operatorname{dim} \operatorname{span}\left(\mathrm{U}_{0}\right)=\left|\mathrm{U}_{0}\right|=\left|\mathrm{V}_{0}\right|=\operatorname{dim} \operatorname{span}\left(\mathrm{V}_{0}\right)$. Having considered that $\mathrm{V}_{0} \subseteq \operatorname{span}\left(\mathrm{U}_{0}\right)$ and both $\mathrm{U}_{0}$ and $\mathrm{V}_{0}$ are finite, we claim $\operatorname{span}\left(\mathrm{U}_{0}\right)=\operatorname{span}\left(\mathrm{V}_{0}\right)$.

Induction: Assume it is true for $p=0,1, \ldots, q-1$. Claim it is also true for $p=q$.

Since $\mathrm{V}_{q} \subseteq \operatorname{span}\left(\bigcup_{i=0}^{q} \mathrm{U}_{i}\right)$, we have

$$
\begin{aligned}
\operatorname{span}\left(\bigcup_{i=0}^{q} \mathrm{U}_{i}\right) & =\operatorname{span}\left(\bigcup_{i=0}^{q} \mathrm{U}_{i} \cup \mathrm{V}_{q}\right) \\
& =\operatorname{span}\left(\bigcup_{i=0}^{q-1} \mathrm{U}_{i} \cup \mathrm{U}_{q} \cup \mathrm{V}_{q}\right) \\
& =\operatorname{span}\left(\bigcup_{i=0}^{q-1} \mathrm{~V}_{i} \cup \mathrm{U}_{q} \cup \mathrm{V}_{q}\right) \\
& \supseteq \operatorname{span}\left(\bigcup_{i=0}^{q} \mathrm{~V}_{i}\right)
\end{aligned}
$$

Notice that $\bigcup_{i=0}^{q} \mathrm{U}_{i} \subseteq\left\{\varepsilon_{0}, \ldots, \varepsilon_{k-1}\right\}$ and $\bigcup_{i=0}^{q} \mathrm{~V}_{i} \subseteq$ $\left\{\varepsilon_{k+1}, \ldots, \varepsilon_{n-1}\right\}$ due to the definition of $\mathrm{U}_{i}$ and $\mathrm{V}_{i}$. By Lemma 4.1, there is no linear dependence in $\bigcup_{i=0}^{q} \mathrm{U}_{i}$ and $\bigcup_{i=0}^{q} \mathrm{~V}_{i}$, which means $\operatorname{dim} \operatorname{span}\left(\bigcup_{i=0}^{q} \mathrm{U}_{i}\right)=\sum_{i=0}^{q}\left|\mathrm{U}_{i}\right|$ $=\sum_{i=0}^{q}\left|\mathrm{~V}_{i}\right|=\operatorname{dim} \operatorname{span}\left(\bigcup_{i=0}^{q} \mathrm{~V}_{i}\right)$. Thus, we have $\operatorname{span}\left(\bigcup_{i=0}^{q} \mathrm{U}_{i}\right)=\operatorname{span}\left(\bigcup_{i=0}^{q} \mathrm{~V}_{i}\right)=\operatorname{span}\left(\bigcup_{i=0}^{q-1} \mathrm{~W}_{i} \cup \mathrm{U}_{q}\right)$ $=\operatorname{span}\left(\bigcup_{i=0}^{q-1} \mathrm{~W}_{i} \cup \mathrm{V}_{q}\right)$, which completes the induction.

Therefore, $\operatorname{span}\left(\bigcup_{i=0}^{\left\lfloor\log _{2} k\right\rfloor} \mathrm{W}_{i}\right)=\operatorname{span}\left(\bigcup_{i=0}^{\left\lfloor\log _{2} k\right\rfloor} U_{i}\right)$. By Lemma 4.1 we know $\bigcup_{i=0}^{\left.\log _{2} k\right\rfloor} U_{i}$ is a basis of $\mathrm{F}_{2}^{k}$. Therefore, $\bigcup_{i=0}^{\left\lfloor\log _{2} k\right\rfloor} \mathrm{W}_{i}$ is also a basis of $\mathrm{F}_{2}^{k}$.

In Theorem 4.1, we consider the symmetric annihilators of Boolean functions in Class 1. We show that all Boolean functions in Class 1 have no symmetric annihilator with degree less than $k$. In Theorem 4.2, we show that all Boolean functions in Class 1 have maximum AI.

Theorem 4.1: Let $n=2 k$ and $f \in \mathrm{SB}_{n}$. If $v_{f}(i)=v_{f}(j)+$ 1 , for any $i, j \in \mathrm{A}_{t}^{k}$ with $0 \leq i<k<j \leq 2 k$ and any 
$1 \leq t \leq\left\lfloor\log _{2} n\right\rfloor$, then there does not exist any nonzero $n$ variable symmetric Boolean function $g$ with degree less than $k$, such that $f g=0$ or $(f+1) g=0$.

Proof: Let $g(x)=\sum_{0<i<k} \lambda_{i} \sigma_{i} \in \mathrm{SB}_{n}$ and $\lambda=\left(\lambda_{0}, \lambda_{1}\right.$, $\left.\ldots, \lambda_{k-1}\right) \in \mathrm{F}_{2}^{k}$. If $f g=0$, then $v_{g}(i)=\varepsilon_{i} \lambda^{\mathrm{T}}=0$ holds when $v_{f}(i)=1$. According to the condition of this theorem that $v_{f}(i)=v_{f}(j)+1$, for any $i, j \in \mathrm{A}_{t}$ with $0 \leq i<k<$ $j \leq 2 k$ and any $1 \leq t \leq\left\lfloor\log _{2} n\right\rfloor$, we can obtain a system of homogeneous linear equations on variables $\lambda_{0}, \ldots, \lambda_{k-1}$ of the form

$$
\left(\begin{array}{c}
\alpha_{0} \\
\alpha_{1} \\
\vdots \\
\alpha_{k-1} \\
\beta
\end{array}\right) \lambda^{\mathrm{T}}=0
$$

where $\alpha_{0}, \alpha_{1}, \ldots, \alpha_{k-1}, \beta \in \mathrm{F}_{2}^{n},\left\{\alpha_{0}, \alpha_{1}, \ldots\right.$, $\left.\alpha_{k-1}\right\}=\bigcup_{i=0}^{\left\lfloor\log _{2} k\right\rfloor} \mathrm{W}_{i}$ and

$$
\beta=\left\{\begin{array}{l}
0, \text { if } v_{f}(k)=0, \\
\varepsilon_{k}, \text { otherwise }
\end{array}\right.
$$

However, by Lemma 4.2, matrices of this kind have full rank. So we have $\lambda=0$; thus, $g=0$.

Denote the system of homogeneous linear equations obtained by the condition $f g=0$ by

$$
\mathrm{M}_{f} \lambda^{\mathrm{T}}=0
$$

i.e., let $\mathrm{M}_{f}$ be the coefficient matrix of the system. Formally, coefficient matrix $M_{f}$ is defined as follows:

$$
M_{f}=\left(\begin{array}{c}
\varepsilon_{i_{1}} \\
\varepsilon_{i_{2}} \\
\vdots \\
\varepsilon_{i_{m}}
\end{array}\right),
$$

where $\varepsilon_{j}$ is a row vector of $M_{f}$ if and only if $v_{f}(j)=1$. The row vectors of $M_{f}$ are ordered by $0 \leq i_{1}<i_{2}<\cdots<i_{m} \leq$ $2 k$.

Similarly, if $(f+1) g=0$, then the coefficient matrix $\mathbf{M}_{f+1}$ of the system of homogeneous linear equations

$$
\mathbf{M}_{f+1} \lambda^{\mathrm{T}}=0
$$

also has full rank. Therefore, $g=0$.

In the following sequel, we only consider the rank of $M_{f}$, which means the order of the row vectors of $M_{f}$ is not important. From the definition of $M_{f}$, we know $\varepsilon_{\omega}$ is a row vector of $M_{f}$ if and only if $v_{f}(\omega)=1$.

Theorem 4.2: Let $n=2 k$ and $f \in \mathrm{SB}_{n}$. If $v_{f}(i)=v_{f}(j)+$ 1 , for any $i, j \in \mathrm{A}_{t}^{k}$ with $0 \leq i<k<j \leq 2 k$ and any $1 \leq t \leq\left\lfloor\log _{2} n\right\rfloor$, then $\mathrm{AI}(f)=k$.

Proof: Assume to the contrary that $\mathrm{AI}(f)<k$. Then, there exists a Boolean function $0 \neq g \in \mathrm{B}_{n}$ with degree less than $k$, such that $f g=0$ or $(f+1) g=0$.

For the case $f g=0$, by Lemma 2.4, there exists a symmetric Boolean function $0 \neq h \in \mathrm{SB}_{n-2 b}$ with $\operatorname{deg}(h) \leq$ $\operatorname{deg}(g)-b<k-b$ for some integer $0 \leq b \leq k$, such that $f h P_{b}=0$. Let $f_{1} \in S B_{n-2 b}$, be defined as

$$
f_{1}\left(x_{2 b+1}, \ldots, x_{n}\right)=f\left(0,1, \ldots, 0,1, x_{2 b+1}, \ldots, x_{n}\right) .
$$

Then

$$
v_{f_{1}}(i)=v_{f}(i+b)
$$

for any $0 \leq i \leq n-2 b$.

i) On one hand, we claim that $f_{1} h=0$. If $f_{1} h \neq 0$, then there exists an $i$ such that $i \in \mathrm{WS}\left(f_{1}\right) \cap \mathrm{WS}(h)$, where $i \in \mathrm{WS}\left(f_{1}\right)$ implies $i+b \in \mathrm{WS}(f)$ by (12) and $i \in$ $\mathrm{WS}(h)$ implies $i+b \in \mathrm{WS}\left(h P_{b}\right)$ by the definition of $P_{b}$. Thus, $i+b \in \mathrm{WS}(f) \cap \mathrm{WS}\left(h P_{b}\right)$, which is contradicted with $f h P_{b}=0$.

ii) On the other hand, we will show a contradiction by proving $f_{1}$ and $f_{1}+1$ do not have symmetric annihilators with degree less than $k-b$. For any $i, j \in A_{t}^{k-b}, 1 \leq t \leq$ $\left\lfloor\log _{2}(n-2 b)\right\rfloor, i<k-b<j$, we have $i+b, j+b \in A_{t}^{k}$ and $i+b<k<j+b$ by Definition 3.2 By the conditions in this theorem, we have $v_{f}(i+b)=v_{f}(j+b)+1$, which implies $v_{f_{1}}(i)=v_{f_{1}}(j)+1$ by (12). Then $f_{1}$ is contained in Class 1. According to Theorem 4.1, $f_{1}$ or $f_{1}+1$ do not have symmetric annihilators with degree less than $k$, which is contradicted with the existence of $h$.

Therefore, $f$ does not have nonzero annihilators with degree less than $k$.

For the case $(f+1) g=0$, we can consider $f_{1}+1$ instead. By the same argument above, we can prove that if $f+1$ does not have nonzero annihilator with degree less than $k$. Therefore, we have $\mathrm{AI}(f)=k$.

\section{Functions in Class 2 Have Maximum Algebraic IMMUNITY}

In this section, we will use the same notations as the last section, such as $\varepsilon_{k}, \mathbf{M}_{f}$, and so on. We always assume $n=2 k$ and $k=\left(k_{m}, \ldots, k_{1}, k_{0}\right)_{2}$, where $m=\left\lfloor\log _{2} k\right\rfloor$. We denote by $\operatorname{supp}(k)=\left\{p \mid k_{p}=1\right\}$, then $m \in \operatorname{supp}(k)$.

We first present Lemmas 5.1, 5.2, 5.3, and 5.4. With these lemmas, we study the annihilator of Boolean functions in Class 2. In Theorem 5.1, the symmetric annihilators of Boolean functions in Class 2 are studied. In Theorem 5.2, all the annihilators of Boolean functions are studied.

The following Lemma plays an important role in the proof of Lemma 5.2.

Lemma 5.1: Given three constants $a, b, c \in \mathrm{F}_{2}$, consider the following system of inequalities on variable $t \in \mathrm{F}_{2}$

$$
\left\{\begin{array}{l}
a \leq b+t \\
t \leq c,
\end{array}\right.
$$

we have

(1) If $a=c$, or $(a, b, c)=(1,1,0)$, then equations (13) have one solution.

(2) If $a=0$ and $c=1$, then equations (13) have two solutions. 
(3) If $(a, b, c)=(1,0,0)$, then equations 13 do not have any solution.

Proof: It is easy to obtain the conclusions.

Lemma 5.2: For any $0 \leq p \leq m+1$, we have

$$
\sum_{j \in \mathrm{A}_{p}, j \preceq k} \varepsilon_{j}=\sum_{j \in \mathrm{A}_{p}, j \preceq k} \varepsilon_{n-j} .
$$

Proof: When $p=0$, equation (14) holds because both sides are $\varepsilon_{k}$. Moreover, if $k_{p-1}=0$, i.e., $p-1 \notin \operatorname{supp}(k)$, then $\left\{j \mid j \in \mathrm{A}_{p}, j \preceq k\right\}=\emptyset$, equation (14) also holds. Therefore, in what follows, we always assume $p \neq 0$ and $k_{p-1}=1$.

For the left-hand side of equation (14), we have

$$
\begin{aligned}
\sum_{j \in \mathrm{A}_{p}, j \preceq k} \varepsilon_{j} & =\sum_{j \in \mathrm{A}_{p}, j \prec k} \sum_{i \preceq j} e_{i} \\
& =\sum_{i \prec k} e_{i} \sum_{j \in \mathrm{A}_{p}, i \preceq j \prec k} 1 .
\end{aligned}
$$

Let $i=\left(i_{m}, \ldots, i_{1}, i_{0}\right)_{2}, j=\left(j_{m}, \ldots, j_{1}, j_{0}\right)_{2}$. By Lemma 3.2, if $j \in \mathrm{A}_{p}$ and $j \preceq k$, then we have

$$
\left(j_{p-1}, j_{p-2}, \ldots, j_{1}, j_{0}\right)=\left(\overline{k_{p-1}}, k_{p-2}, \ldots, k_{1}, k_{0}\right) .
$$

Therefore,

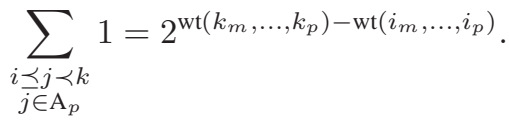

Thus

$$
\sum_{\substack{i \preceq j \prec k \\ j \in \mathrm{A}_{p}}} 1=1
$$

if and only if

$$
i=\left(k_{m}, \ldots, k_{p}, \overline{k_{p-1}}, i_{p-2}, \ldots, i_{1}, i_{0}\right)_{2}
$$

with $\left(i_{p-2}, \ldots, i_{1}, i_{0}\right) \preceq\left(k_{p-2}, \ldots, k_{1}, k_{0}\right)$. Hence, we have

$$
\sum_{j \in \mathrm{A}_{p}, j \preceq k} \varepsilon_{j}=\sum_{\substack{i=\left(k_{m}, \ldots, k_{p}, \overline{k_{p-1}}, i_{p-2}, \ldots, i_{1}, i_{0}\right)_{2} \\\left(i_{p-2}, \ldots, i_{1}, i_{0}\right) \preceq\left(k_{p-2}, \ldots, k_{1}, k_{0}\right)}} e_{i} .
$$

For the right-hand side of equation (14), we have

$$
\begin{aligned}
\sum_{\substack{j \prec k, j \in \mathrm{A}_{p}}} \varepsilon_{2 k-j} & =\sum_{\substack{j \prec k, j \in \mathrm{A}_{p}}} \sum_{\substack{i \preceq 2 k-j \\
0 \leq i \leq k-1}} e_{i} \\
& =\sum_{0 \leq i \leq k-1} e_{i} \sum_{\substack{i \preceq 2 k-j \\
j \prec k, j \in \mathrm{A}_{p}}} 1 .
\end{aligned}
$$

If $j \in \mathrm{A}_{p}$, then the last $p$ bits of $j$ and $2 k-j$ are both $\left(\overline{k_{p-1}}\right.$, $\left.k_{p-2}, \ldots, k_{1}, k_{0}\right)$. Hence,

$$
k-j=\left(k_{m}+j_{m}, \ldots, k_{p}+j_{p}, 1,0, \ldots, 0\right)_{2}
$$

and we can write $2 k-j=\left(k_{m}+s_{m}, k_{m-1}+j_{m}+s_{m-1}, \ldots\right.$, $\left.k_{p+1}+j_{p+2}+s_{p+1}, k_{p}+j_{p+1}, \overline{j_{p}}, 0, k_{p-2}, \ldots, k_{0}\right)_{2}$, where for any $q>p, s_{q}=1$ if and only if $\left(k_{q-1}, j_{q}, s_{q-1}\right)=(1,1,1)$ or, $k_{q-1}=0$ and $\left(j_{q}, s_{q-1}\right) \neq(0,0)$. Note that the additions are in $\mathrm{F}_{2}$.

If

$$
\left(i_{p-1}, i_{p-2}, \ldots, i_{1}, i_{0}\right) \npreceq\left(\overline{k_{p-1}}, k_{p-2}, \ldots, k_{1}, k_{0}\right),
$$

then $\left\{j \mid i \preceq 2 k-j, j \prec k, j \in \mathrm{A}_{p}\right\}=\emptyset$; thus,

$$
\sum_{\substack{i \preceq 2 k-j \\ j \prec k, j \in \mathrm{A}_{p}}} 1=0 .
$$

Now assume

$$
\left(i_{p-1}, i_{p-2}, \ldots, i_{1}, i_{0}\right) \preceq\left(\overline{k_{p-1}}, k_{p-2}, \ldots, k_{1}, k_{0}\right),
$$

then $i \preceq 2 k-j, j \prec k, j \in \mathrm{A}_{p}$ if and only if

$$
\left(j_{p-1}, j_{p-2}, \ldots, j_{0}\right)=\left(\overline{k_{p-1}}, k_{p-2}, \ldots, k_{0}\right)
$$

and

$$
\left\{\begin{array}{l}
\left(i_{m}, \ldots, i_{p}\right) \preceq\left(k_{m-1}+j_{m}+s_{m-1}, \ldots, k_{p}+j_{p+1}, \overline{j_{p}}\right), \\
\left(j_{m}, \ldots, j_{x}\right) \preceq\left(k_{m}, \ldots, k_{p}\right) .
\end{array}\right.
$$

Equations 15 are equivalent to the intersections of a series of systems of inequalities as follows:

$$
\begin{gathered}
\left\{\begin{array}{l}
i_{p} \leq 1+j_{p} \\
j_{p} \leq k_{p}
\end{array},\left\{\begin{array}{l}
i_{p+1} \leq k_{p}+j_{p+1} \\
j_{p+1} \leq k_{p+1}
\end{array}, \ldots,\right.\right. \\
\left\{\begin{array}{l}
i_{m} \leq k_{m-1}+j_{m}+s_{m-1} \\
j_{m} \leq k_{m}
\end{array}\right.
\end{gathered}
$$

If $\left(i_{m}, \ldots, i_{p}\right)=\left(k_{m}, \ldots, k_{p}\right)$, by Lemma 5.1 , each system of inequalities has one and only one solution with respect to $j_{q}$. Thus, we can conclude that there is one and only one solution of $\left(j_{m}, \ldots, j_{0}\right)_{2}$ satisfying $i \preceq 2 k-j, j \prec k, j \in \mathrm{A}_{p}$. Therefore, the coefficient of $e_{i}$, where $i=\left(k_{m}, \ldots, k_{p}, \overline{k_{p-1}}\right.$, $\left.i_{p-2}, \ldots, i_{1}, i_{0}\right)_{2}$, equals 1 .

Else if $\left(i_{m}, \ldots, i_{p}\right) \neq\left(k_{m}, \ldots, k_{p}\right)$, then the set $\left\{q \mid k_{q}=\right.$ $\left.1, i_{q}=0\right\}$ is not empty. For any such $q$, the corresponding system of inequalities with respect to $j_{q}$ has two solutions, according to Lemma 5.1. While for any other systems, either one solution or no solution exists. Therefore, the number of $j$ 's satisfying $i \preceq 2 k-j, j \prec k$, and $j \in \mathrm{A}_{p}$ is even. Therefore, the coefficient of $e_{i}$ equals 0 . As a result, we have

$$
\sum_{j \prec k, j \in \mathrm{A}_{p}} \varepsilon_{2 k-j}=\sum_{\substack{i=\left(k_{m}, \ldots, k_{p}, \overline{k_{p-1}}, i_{p-2}, \ldots, i_{1}, i_{0}\right)_{2} \\\left(i_{p-2}, \ldots, i_{1}, i_{0}\right) \preceq\left(k_{p-2}, \ldots, k_{1}, k_{0}\right)}} e_{i} .
$$

So equation (14) holds. This finishes the proof of this theorem.

Lemma 5.3: For any positive integer $k$, we have

$$
\varepsilon_{k}=\sum_{j \prec k} \varepsilon_{j} .
$$

Proof: Let $i=\left(i_{m}, \ldots, i_{0}\right)_{2}, j=\left(j_{m}, \ldots, j_{0}\right)_{2}$ and $k=$ $\left(k_{m}, \ldots, k_{0}\right)_{2}$. Consider the equation

$$
\sum_{j \preceq k} \varepsilon_{j}=\sum_{j \preceq k} \sum_{\substack{i \preceq j \\ 0 \leq i \leq k-1}} e_{i}=\sum_{0 \leq i \leq k-1} e_{i} \sum_{i \preceq j \preceq k} 1 .
$$

Notice here, $i \neq k$. By the definition of $\preceq$, for each $i \preceq k$, the number of $j$ such that $i \preceq j \preceq k$ is

$$
\sum_{i \preceq j \preceq k} 1=2^{\mathrm{wt}\left(k_{m}, \ldots, k_{0}\right)-\mathrm{wt}\left(i_{m}, \ldots, i_{0}\right)} .
$$


Then, we have $\sum_{i \prec j \prec k} 1$ is always even because $i \neq k$, which means $\sum_{j \preceq k} \varepsilon_{j}=0$. Thus, we have $\varepsilon_{k}=\sum_{j \prec k} \varepsilon_{j}$.

Lemma 5.4: For any positive integer $k$, we have

$$
\varepsilon_{k}=\sum_{j \prec k} \varepsilon_{n-j} .
$$

Proof: According to Lemma 5.3, we have

$$
\varepsilon_{k}=\sum_{j \prec k} \varepsilon_{j}=\sum_{0 \leq p \leq m+1} \sum_{j \in A_{p}, j \prec k} \varepsilon_{j}
$$

because $A_{p}$ for all $0 \leq p \leq m+1$ is a partition of $\{0,1, \ldots, n\}$. By applying Lemma 5.2 to the right-hand side of the equation above, we have

$$
\varepsilon_{k}=\sum_{0 \leq p \leq m+1} \sum_{j \in A_{p}, j \prec k} \varepsilon_{n-j}=\sum_{j \prec k} \varepsilon_{n-j} .
$$

With these lemmas above, we have

Theorem 5.1: Suppose $f \in \mathrm{SB}_{n}$ such that there exists a function $f^{\prime}$ in Class 1 and an integer $t \prec^{\prime} k$ such that

$$
\begin{aligned}
& v_{f}=v_{f^{\prime}}+e_{t}+\delta e_{k}, \text { or } \\
& v_{f}=v_{f^{\prime}}+e_{n-t}+\delta^{\prime} e_{k},
\end{aligned}
$$

where

$$
\delta=v_{f^{\prime}}(t)+v_{f^{\prime}}(k)
$$

and

$$
\delta^{\prime}=v_{f^{\prime}}(n-t)+v_{f^{\prime}}(k) .
$$

Then both $f$ and $f+1$ have no nonzero symmetric annihilators with degree less than $k$.

Proof: Any $n$-variable symmetric Boolean function $g$ with degree less than $k$ can be written as $g=\sum_{0<i<k} \lambda_{i} \sigma_{i}$. If $f g=0$ or $(f+1) g=0$, similar to the proof of Theorem 4.1 , we can set up a system of homogeneous linear equations on variables $\lambda_{i}$ 's. If we can show that the coefficient matrix $M_{f}$ has full rank, then the equation system has only zero solution which means $\lambda_{g}=0$; thus, $g=0$. By the definition of coefficient matrix 111), $M_{f}$ is only slightly different from that of equation $M_{f^{\prime}}$, because $v_{f}(i)=v_{f^{\prime}}(i)$ holds except for $i=t, k, n-t$. Notice that we only care about the rank of $M_{f}$ and $M_{f^{\prime}}$. Since $f^{\prime}$ is in Class 1 , we have $v_{f^{\prime}}(t)=v_{f^{\prime}}(n-t)+1$ and $M_{f^{\prime}}, M_{f^{\prime}+1}$ both have full rank by the proof of Theorem 4.1 Let $a \in \mathrm{F}_{2}$, if

$$
\left(v_{f^{\prime}}(t), v_{f^{\prime}}(k), v_{f^{\prime}}(n-t)\right)=(a, a, a+1),
$$

then

$\left(v_{f}(t), v_{f}(k), v_{f}(n-t)\right)=(a+1, a, a+1)$ or $(a, a+1, a)$.

Case 1: $a=0$.

$$
\left(v_{f^{\prime}}(t), v_{f^{\prime}}(k), v_{f^{\prime}}(n-t)\right)=(0,0,1) .
$$

Let $p_{1}$ be the integer satisfying $t \in A_{p_{1}}$, then for any $i>k$ and $i \in A_{p_{1}}$, we have $v_{f^{\prime}}(i)=1$ for the reason $f^{\prime}$ belongs to Class 1. Consider the difference between the coefficient matrix $\mathrm{M}_{f}$ and $M_{f^{\prime}}$. By the definition of coefficient matrix [11), since $v_{f^{\prime}}(n-t)=1$ and $v_{f^{\prime}}(t)=v_{f^{\prime}}(k)=0, \varepsilon_{n-t}$ is a row vector of $M_{f^{\prime}}$ but $\varepsilon_{t}$ and $\varepsilon_{k}$ are not.

When $\left(v_{f}(t), v_{f}(k), v_{f}(n-t)\right)=(1,0,1)$, the row vectors of $M_{f}$ and $M_{f^{\prime}}$ are all the same except that an extra $\varepsilon_{t}$ is a row vector of $M_{f}$ by the definition of coefficient matrix (11). Then, $M_{f}$ has full rank as $M_{f^{\prime}}$ has full rank.

When $\left(v_{f}(t), v_{f}(k), v_{f}(n-t)\right)=(0,1,0)$, the only difference with $M_{f^{\prime}}$ is that $\varepsilon_{k}$ is a row vector of $M_{f}$ but $\varepsilon_{n-t}$ is not by the definition of coefficient matrix (111). If we can prove that $\varepsilon_{n-t}$ is a linear combination of $\varepsilon_{k}$ and other row vectors in $M_{f}$, then $M_{f}$ also has full rank. In the next paragraph, we will complete this proof.

By Lemma 5.4 and the fact that $A_{p}$ for all $0 \leq p \leq m+1$ is a partition of $\{0,1, \ldots, n\}$, we have

$$
\sum_{j \in A_{p_{1}}, j \preceq k} \varepsilon_{n-j}=\varepsilon_{k}+\sum_{\substack{p \neq p_{1} \\ 0 \leq p \leq m+1}} \sum_{\substack{j \in A_{p} \\ j \prec k}} \varepsilon_{n-j} .
$$

Since $t \in A_{p_{1}}$ and $t \prec^{\prime} k$ which means $t \preceq k$, then $\varepsilon_{n-t}$ appears on the left-hand side of (16). Remember that for any $i>k$ where $i \in A_{p_{1}}$, we have $v_{f^{\prime}}(i)=1$. Thus, we have $v_{f}(i)=1$ for any such $i$ with $i \neq n-t$, which means all vectors in the left-hand side of (16) are row vectors of $M_{f}$ except $\varepsilon_{n-t}$ by the definition of (12). While for the righthand side of (16), notice that since $f^{\prime}$ is contained in Class 1. Then if one element $\omega$ in the set $\left\{j \mid j \in A_{p}, j \prec k\right\}$ (or $\left\{n-i \mid j \in A_{p}, j \prec k\right\}$ ) satisfies $v_{f^{\prime}}(\omega)=1$, so do the other elements in the set. By the definition of 111), if one term in $\sum_{j \in A_{p}, j \prec k} \varepsilon_{j}$ ( or $\sum_{j \in A_{p}, j \prec k} \varepsilon_{n-j}$ ) is a row vector of $M_{f^{\prime}}$, so are the other terms. By the definition of $M_{f}$, we can see $M_{f}$ also has this property as $M_{f^{\prime}}$ for any $p \neq p_{1}$. Then, we can turn all terms on the right-hand side of (16) into the row vectors of $M_{f}$ by Lemma 5.2 as follows. If $\sum_{j \in A_{p}, j \prec k} \varepsilon_{n-j}$ with $p \neq p_{1}$ appears but not row vectors of $M_{f}$, then we apply Lemma 5.2 to replace $\sum_{j \in A_{p}, j \prec k} \varepsilon_{n-j}$ by $\sum_{j \in A_{p}, j \prec k} \varepsilon_{j}$. We can continue this procedure until that any vector that appears in the right-hand side also appears as a row vector of $\mathbf{M}_{f}$.

After this, both sides of (16) become row vectors of $M_{f^{\prime}}$ except $\varepsilon_{n-t}$. Thus we can conclude that $\varepsilon_{n-t}$ is a linear combination of row vectors of $\mathrm{M}_{f}$. Therefore, $\mathrm{M}_{f}$ has full rank because $M_{f^{\prime}}$ has full rank.

Consider the difference between $\mathbf{M}_{f+1}$ and $\mathbf{M}_{f^{\prime}+1}$. Since

$$
\left(v_{f^{\prime}+1}(t), v_{f^{\prime}+1}(k), v_{f^{\prime}+1}(n-t)\right)=(1,1,0),
$$

and recalling the definition of coefficient matrix 111), $\varepsilon_{t}$ and $\varepsilon_{k}$ are row vectors of $\mathbf{M}_{f^{\prime}+1}$. While for $\mathbf{M}_{f+1}$, if $\left(v_{f+1}(t), v_{f+1}(k), v_{f+1}(n-t)\right)=(0,1,0)$, the only difference is that $\varepsilon_{t}$ is not a row vector of $M_{f+1}$. By the same argument above using Lemmas 5.2 and 5.3, $\varepsilon_{t}$ is a linear combination of row vectors of $\mathrm{M}_{f+1}$. This has no influence to the rank of $\mathbf{M}_{f+1}$. Therefore, $\mathbf{M}_{f+1}$ also has full rank because $M_{f^{\prime}+1}$ has full rank.

Otherwise, if $\left(v_{f+1}(t), v_{f+1}(k), v_{f+1}(n-t)\right)=(1,0,1)$, according to the definition of coefficient matrix (11), the difference is that $\varepsilon_{n-t}$ is a row vector of $M_{f+1}$ but $\varepsilon_{k}$ is not. This matrix is also full rank because $\varepsilon_{k}$ is a linear combination of other row vectors in $M_{f^{\prime}}$ due to Lemma 4.2.

Case 2: $a=1$. 
We can reach the conclusion that the matrices $M_{f}$ and $M_{f+1}$ always have full rank in the same way of Case 1.

Else if

$$
\left(v_{f^{\prime}}(t), v_{f^{\prime}}(k), v_{f^{\prime}}(n-t)\right)=(a, a+1, a+1),
$$

then

$\left(v_{f}(t), v_{f}(k), v_{f}(n-t)\right)=(a+1, a, a+1)$ or $(a, a+1, a)$.

In a similar way to the discussion as above, no matter $a=0$ or 1 and no matter what $f^{\prime}$ is, the coefficient matrices $\mathbf{M}_{f}$ and $\mathbf{M}_{f+1}$ always have full rank, which implies $\lambda=0$; thus, $g=0$.

Therefore, both $f$ and $f+1$ have no symmetric annihilators with degree less than $k$.

Theorem 5.2: Suppose $f \in \mathrm{SB}_{n}$ such that there exists a function $f^{\prime}$ in Class 1 and an integer $t \prec^{\prime} k$, such that

$$
\begin{aligned}
& v_{f}=v_{f^{\prime}}+e_{t}+\delta e_{k}, \text { or } \\
& v_{f}=v_{f^{\prime}}+e_{n-t}+\delta^{\prime} e_{k},
\end{aligned}
$$

where

$$
\delta=v_{f^{\prime}}(t)+v_{f^{\prime}}(k)
$$

and

$$
\delta^{\prime}=v_{f^{\prime}}(n-t)+v_{f^{\prime}}(k)
$$

Then $\mathrm{AI}(f)=k$.

Proof: Assume to the contrary that $\mathrm{AI}(f)<k$. Then, there exists a Boolean function $0 \neq g \in \mathrm{B}_{n}$ with degree less than $k$, such that $f g=0$ or $(f+1) g=0$.

For the case $f g=0$, by Lemma 2.4, there exists a symmetric Boolean function $0 \neq h \in \operatorname{SB}_{n-2 b}$ with $\operatorname{deg}(h)$ $\leq \operatorname{deg}(g)-b<k-b$ for some integer $0 \leq b \leq k$, such that $f h P_{b}=0$.

Let $f_{1} \in S B_{n-2 b}$ be defined as

$$
f_{1}\left(x_{2 b+1}, \ldots, x_{n}\right)=f\left(0,1, \ldots, 0,1, x_{2 b+1}, \ldots, x_{n}\right) .
$$

Then

$$
v_{f_{1}}(i)=v_{f}(i+b)
$$

for any $0 \leq i \leq n-2 b$.

On one hand, by following the same argument of i) in the proof of Theorem 4.2 we have $f_{1} h=0$ because $f h P_{b}=0$.

On the other hand, we will show a contradiction by proving $f_{1}$ and $f_{1}+1$ do not have symmetric annihilators with degree less than $k-b$. For any $i, j \in A_{p}^{k-b}, 1 \leq p \leq\left\lfloor\log _{2}(n-2 b)\right\rfloor$, $i<k-b<j$, we have $i+b, j+b \in A_{p}^{k}$ and $i+b<k<j+b$ by Definition 3.2

If $t<b$, then $t-b, n-(t-b) \notin A_{p}^{k-b}$ for any $1 \leq p \leq$ $\left\lfloor\log _{2}(n-2 b)\right\rfloor$. Then, by the conditions of this theorem, we have $v_{f}(i+b)=v_{f}(j+b)+1$, which implies $v_{f_{1}}(i)=v_{f_{1}}(j)+$ 1 by (17). Then, $f_{1}$ is contained in Class 1. According to Theorem 4.1, $f_{1}$ and $f_{1}+1$ do not have symmetric annihilators with degree less than $k$, which contradicts the existence of $h$.

If $t \geq b$, then $t-b \prec^{\prime} k-b$ by the definition of $\prec^{\prime}$ and $t-b$, $n-(t-b) \in A_{p}^{k-b}$ for some $1 \leq p \leq\left\lfloor\log _{2}(n-2 b)\right\rfloor$. Then, by the conditions of this theorem, we have $v_{f}(t)=v_{f}(n-t)=$ $v_{f}(k)+1$ and $v_{f}(i+b)=v_{f}(j+b)+1$ for $i+b \neq t$ and $j+b \neq$ $n-t$, which implies $v_{f_{1}}(t-b)=v_{f_{1}}(n-(t-b))=v_{f_{1}}(k)+1$ and $v_{f_{1}}(i)=v_{f_{1}}(j)+1$ for $i \neq t-b$ and $j \neq n-(t-b)$. Then, $f_{1}$ is contained in Class 2. According to Theorem 5.1, $f_{1}$ and $f_{1}+1$ do not have symmetric annihilators with degree less than $k$, which contradicts the existence of $h$.

Therefore, $f$ does not have nonzero annihilators with degree less than $k$.

For the case $(f+1) g=0$, we can consider $f_{1}+1$ instead. By the same argument above, we can prove that if $f+1$ does not have nonzero annihilator with degree less than $k$. Therefore, we have $\mathrm{AI}(f)=k$.

\section{MAIN RESUlT}

Finally, we obtain the following main result.

Theorem 6.1: Let $n=2 k, k=\left(k_{m}, \ldots, k_{1}, k_{0}\right)_{2}$, where $m=\left\lfloor\log _{2} k\right\rfloor$. Let $\operatorname{supp}(k)=\left\{p \mid k_{p}=1\right\}$. Given $f \in \mathrm{SB}_{n}$, then $\mathrm{AI}(f)=k$ if and only if $v_{f}$ satisfies one of the following three cases:

1) There exist $a_{0}, a_{1}, a_{2}, \ldots, a_{m}, a_{m+1} \in \mathrm{F}_{2}$, such that for any $1 \leq t \leq m+1$ and $i, j \in A_{t}, 0 \leq i<k<j \leq$ $2 k, v_{f}(i)=v_{f}(j)+1=a_{t}$ holds, and $v_{f}(k)=a_{0}$.

2) There exist $a_{1}, a_{2}, \ldots, a_{m}, a_{m+1} \in \mathrm{F}_{2}$, such that for any $1 \leq t \leq m$ and $i, j \in A_{t}, 0 \leq i<k<j \leq 2 k, v_{f}(i)=$ $v_{f}(j)+1=a_{t}$ holds, and

$\left.\left(v_{f}\left(k-2^{m}\right), v_{f}(k), v_{f}\left(k+2^{m}\right)\right)\right)=\left(a_{m+1}, a_{m+1}+1, a_{m+1}\right) ;$

3) There exists an integer $p_{0} \in \operatorname{supp}(k), p_{0} \neq m$, and $a_{1}$, $a_{2}, \ldots, a_{m}, a_{m+1}, b_{p_{0}} \in \mathrm{F}_{2}$ such that for any $1 \leq t \leq m+1$, $i, j \in A_{t}, 0 \leq i<k<j \leq 2 k, i \neq i_{0}$ and $j \neq n-i_{0}$, $v_{f}(i)=v_{f}(j)+1=a_{t}$ holds, and

$$
\begin{gathered}
\left(v_{f}\left(i_{0}\right), v_{f}(k), v_{f}\left(n-i_{0}\right)\right)=\left(b_{p_{0}}, \overline{b_{p_{0}}}, b_{p_{0}}\right), \text { or } \\
\left(v_{f}\left(i_{0}\right), v_{f}(k), v_{f}\left(n-i_{0}\right)\right)=\left(\overline{b_{p_{0}}}, b_{p_{0}}, \overline{b_{p_{0}}}\right),
\end{gathered}
$$

where $i_{0}=\left(k_{p_{0}-1}, \ldots, k_{1}, k_{0}\right) \prec^{\prime} k$.

The total number is $(2 \mathrm{wt}(n)+1) 2^{\left\lfloor\log _{2} n\right\rfloor}$. And the values of their Hamming weight are $\left\{2^{n-1} \pm \frac{1}{2}\left(\begin{array}{c}n \\ n / 2\end{array}\right), 2^{n-1}+\frac{1}{2}\left(\begin{array}{c}n \\ n / 2\end{array}\right)-\left(\begin{array}{c}n \\ i\end{array}\right)\right.$, $2^{n-1}-\frac{1}{2}\left(\begin{array}{c}n \\ n / 2\end{array}\right)+\left(\begin{array}{c}n \\ i\end{array}\right)$, for any $\left.i \prec^{\prime} k\right\}$.

Proof: Notice that $v_{f}$ satisfies item 1 if and only if $f$ belongs to Class $1, v_{f}$ satisfies items 2 or 3 if and only if $f$ belongs to Class 2 . Then, the necessity is proved by Theorem 3.5, while the sufficiency is proved by Theorems 4.2 and 5.2.

The number of functions satisfying item 1 is $2^{m+2}$, the number of functions satisfying item 2 is $2^{m+1}$, and the number of functions satisfying item 3 is $(\operatorname{wt}(n)-1) 2^{m+2}$, because different choices of $a_{i}$ 's, $b_{p_{0}}$ and $i_{0}$ will generate different functions. Therefore, the total number is $(2 \mathrm{wt}(n)+1) 2^{m+1}$. The corresponding values of Hamming weight are also easy to calculate.

For example, when $n=14$, we have $k=7, m=\left\lfloor\log _{2} 7\right\rfloor=$ 2 and $\operatorname{supp}(7)=\{0,1,2\}$.

There are 16 functions satisfying the conditions of item 1). Among them, the simplified value vectors of those satisfying $v_{f}(7)=1$ are as follows: 


\begin{tabular}{c|c}
\hline$f$ & SVV: $v_{f}(0) \ldots v_{f}(14)$ \\
\hline 1 & 000000011111111 \\
\hline 2 & 000100011110111 \\
\hline 3 & 010001011011101 \\
\hline 4 & 101010110101010 \\
\hline 5 & 010101011010101 \\
\hline 6 & 101110110100010 \\
\hline 7 & 111011110001000 \\
\hline 8 & 111111110000000 \\
\hline
\end{tabular}

All of them have the same Hamming weight $2^{13}+\frac{1}{2}\left(\begin{array}{c}14 \\ 7\end{array}\right)=9908$, whereas all of their complements have the same Hamming weight $2^{13}-\frac{1}{2}\left(\begin{array}{c}14 \\ 7\end{array}\right)=6476$.

There are 8 functions satisfying the conditions of item 2 . Among them, the simplified value vectors of those satisfying $v_{f}(7)=1$ are as follows:

\begin{tabular}{c|c}
\hline$f$ & $\mathrm{SVV}: v_{f}(0) \ldots v_{f}(14)$ \\
\hline 1 & 000000011110111 \\
\hline 2 & 010001011010101 \\
\hline 3 & 101010110100010 \\
\hline 4 & 111011110000000 \\
\hline
\end{tabular}

All of them have the same Hamming weight $2^{13}+\frac{1}{2}\left(\begin{array}{c}14 \\ 7\end{array}\right)$ $\left(\begin{array}{c}14 \\ 3\end{array}\right)=9544$, whereas all of their complements have the same Hamming weight $2^{13}-\frac{1}{2}\left(\begin{array}{c}14 \\ 7\end{array}\right)+\left(\begin{array}{c}14 \\ 3\end{array}\right)=6840$.

There are 32 functions satisfying the conditions of item 3 because $\operatorname{wt}(14)=3$ and $i_{0}$ can be 0 or 1 . When $i_{0}=0$, among such functions, the simplified value vectors of those satisfying $v_{f}(7)=1$ are as follows:

\begin{tabular}{c|c}
\hline$f$ & $\mathrm{SVV}: v_{f}(0) \ldots v_{f}(14)$ \\
\hline 1 & 000000011111110 \\
\hline 2 & 010001011011100 \\
\hline 3 & 001010110101010 \\
\hline 4 & 011011110001000 \\
\hline 5 & 000100011110110 \\
\hline 6 & 010101011010100 \\
\hline 7 & 001110110100010 \\
\hline 8 & 011111110000000 \\
\hline
\end{tabular}

All of them have the same Hamming weight $2^{13}+\frac{1}{2}\left(\begin{array}{c}14 \\ 7\end{array}\right)$ $\left(\begin{array}{c}14 \\ 0\end{array}\right)=9907$, whereas all of their complements have the same Hamming weight $2^{13}-\frac{1}{2}\left(\begin{array}{c}14 \\ 7\end{array}\right)+\left(\begin{array}{c}14 \\ 0\end{array}\right)=6477$.

When $i_{0}=1$, among such functions, the simplified value vectors of those satisfying $v_{f}(7)=1$ are as follows:

\begin{tabular}{c|c}
\hline$f$ & $\mathrm{SVV}: v_{f}(0) \ldots v_{f}(14)$ \\
\hline 1 & 000000011111101 \\
\hline 2 & 000001011011101 \\
\hline 3 & 101010110101000 \\
\hline 4 & 101011110001000 \\
\hline 5 & 000100011110101 \\
\hline 6 & 000101011010101 \\
\hline 7 & 101110110100000 \\
\hline 8 & 101111110000000 \\
\hline
\end{tabular}

All of them have the same Hamming weight $2^{13}+\frac{1}{2}\left(\begin{array}{c}14 \\ 7\end{array}\right)$ $\left(\begin{array}{c}14 \\ 1\end{array}\right)=9894$, whereas all their complements have the same Hamming weight $2^{13}-\frac{1}{2}\left(\begin{array}{c}14 \\ 7\end{array}\right)+\left(\begin{array}{c}14 \\ 1\end{array}\right)=6490$.

\section{CONCLUSION}

In this paper, we give a necessary and sufficient condition for an even-variable symmetric Boolean function to reach maximum algebraic immunity for the first time.
We first study the weight supports of low-degree symmetric Boolean functions and use some linear algebras to obtain some necessary conditions for an even-variable symmetric Boolean function to reach maximum algebraic immunity, then we divide the functions satisfying these conditions into two classes. Finally, we proved that functions of either class indeed have maximum algebraic immunity. Thus, the problem of finding all even-variable symmetric Boolean functions with maximum algebraic immunity is solved.

\section{ACKNOWLEDGMENT}

The authors are grateful to the anonymous reviewers for their valuable comments and kind suggestions that have improved the technical quality and editorial presentation of this paper.

\section{REFERENCES}

[1] F. Armknecht, "Improving fast algebraic attacks," in Proc. Workshop on Fast Software Encryption 2004 (Lecture Notes in Computer Science). Berlin, Germany: Springer-Verlag, 2004, vol.3017, pp. 65-82.

[2] A. Braeken, "Cryptographic properties of Boolean functions and Sboxes," Ph.D. dissertation, Dept. Electr. Eng. (ESAT), Katholieke Univ., Luven,Belgium, 2006.

[3] A. Braeken and B. Preneel, "On the algebraic immunity of symmetric Boolean functions," Indocrypt 2004(Lecture Notes in Computer Science). Berlin Germany: Springer-verlag, 2005, vol. 3797, pages 35-48.

[4] A. Canteaut and M. Videau, "Symmetric boolean functions," IEEE Trans. Inf. Theory, vol.51, no.8, pp.2791-2811, AUGUST 2005.

[5] C. Carlet, P. Charpin, and V. Zinoviev, "Codes, bent functions and permutations suitable for DES-like cryptosystems," Designs, Codes, Cryptogr. vol.15, pp. 125-156, 1998.

[6] N. T. Courtois and W. Meier, "Algebraic attacks on stream ciphers with linear feed back," in Advances in Cryptology - Eurocrypt 2003 (Lecture Notes in Computer Scinece). Berlin, Germany: Springer-Verlag, 2003, vol. 2656, pp. 345-359.

[7] D. K. Dalai, K. C. Gupta and S. Maitra, "Results on algebraic immunity for cryptographically significant Boolean functions," in INDOCRYPT 2004 (Lecture Notes in Computer Science). Berlin, Germany: SpringerVerlag, 2004, vol. 3348, pp. 92-106.

[8] D. K. Dalai, K. C. Gupta, and S. Maitra, "Cryptographically significant Boolean functions: Construction and analysis in terms of algebraic immunity," in Workshop on Fast Software Encryption (FSE 2005) (Lecture Notes in Computer Science). Berlin, Germany: Springer-Verlag, 2005, vol. 3557, pp. 98-111.

[9] D. K.Dalai, S. Maitra and S. Sarkar, "Basic theory in construction of Boolean functions with maximum possible annihilator immunity," Des. Codes Cryptogr., vol.40, no.1, pp.41-58, Jul.2006.

[10] K. Q. Feng, Q. Y. Liao and F. Liu, "On $2^{m}$-variable symmetric Boolean functions with maximum algebraic immunity $2^{m-1}$." Preprint.

[11] F. Liu and K. Q. Feng, "Efficient computation of algebraic immunity of symmetric Boolean functions," in Theory and Applications of Models of Computation 2007 (Lecture Notes in Computer Science). Berlin Germany: Springer-Verlag, 2007, vol. 4484, pp. 318-329.

[12] Q. Y.Liao, F. Liu and K. Feng, "On $\left(2^{m}+1\right)$-variable symmetric Boolean functions with submaximum algebraic immunity $2^{m-1}$, , in Science in China. Series A. Jan. 2009, vol.52, no.1, 17-28.

[13] N. Li, and W. F. Qi, "Symmetric Boolean functions depending on an odd number of variables with maximum algebraic immunity," in IEEE Trans. Inf. Theory, vol. 52, no.5, pp. 2271-2273, MAY 2006.

[14] W. Meier,E. Pasalic and C. Carlet, "Algebraic attacks and decomposition of Boolean functions," in Advances in Cryptology - EUROCRYPT 2004 (Lecture Notes in Computer Science). Berlin, Germany: Springer-Verlag, 2004, vol. 3027, pp. 474-491.

[15] L. J. Qu, K. Feng, F. Liu, and L. Wang, "Constructing symmetric Boolean functions with maximum algebraic immunity," IEEE Trans. Inf. Theory, vol.55, no 5, pp. 2406-2412, May.2009.

[16] L. J. Qu, C. Li and K. Feng, "A note on symmetric Boolean functions with maximum algebraic immunity in odd number of variables," IEEE Trans. Inf. Theory, vol. 53, no. 8, pp. 2908-2910, Aug. 2007. 
[17] L. J. Qu and C. Li, "On the $2^{m}$-variable symmetric Boolean function with maximum algebraic immunity," in Science in China. Series F. Feb.2008, vol.51, no.2, 120-127.

[18] S. Rønjom and T. Helleseth, "A new attack on filter generator," IEEE Trans. Inf. Theory, vol.53, no. 5, pp. 1752-1758, may. 2007.

[19] I. Wegener, "The Complexity of Boolean Functions," New York: Wiley, 1987.

[20] R. M. Wilson, "A diagonal form for the incidence matrices of t-subsets vs k-subsets," Eur.J.Combin, vol.11, pp.609-614, 1990.

[21] W. Zhang and R. Li, "Algebraic Immunity Of Even Variable Symmetric Boolean Functions," in WKDD 2009. pp. 559-561, Jan. 2009.

\begin{tabular}{|c|}
\hline \\
\\
PLACE \\
PHOTO \\
HERE \\
\end{tabular}

Hui Wang was born in Anhui, China in 1988. He is currently working towards the Ph.D. degree at Fudan University, China. 